\title{
Permanent deformations and strains in a shear building excited by a strong motion pulse
}

\author{
V. Gicev ${ }^{\mathrm{a}}$, M.D. Trifunac ${ }^{\mathrm{b}, *}$

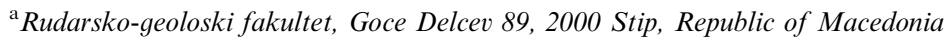 \\ ${ }^{\mathrm{b}}$ Department of Civil Engineering, University of Southern California, Los Angeles, CA 90089, USA
}

Accepted 31 May 2006

\begin{abstract}
Examples of non-linear wave propagation in an elasto-plastic building are presented for excitation by pulses of strong ground motion characteristic of the near-field shaking near earthquake faults. Conditions that lead to the occurrence of permanent deformations in the building are investigated, and the amplitudes and wavelengths of incident pulses that lead to non-linear response are shown. Because the building can fail during the first passage of the incident wave pulse up and down the building (during a period that is shorter than the first natural period of the building), it is concluded that for the analysis and the design of structures in the near-field of earthquake shaking the wave propagation method of analysis must be used in place of the response spectrum method, which is based on the vibrational solution of the same governing equations.
\end{abstract}

(C) 2007 Elsevier Ltd. All rights reserved.

\section{Introduction}

Rational design of earthquake-resistant structures requires realistic representation of the problem - that is, wise selection of the mathematical model and the associated differential equations, which are the tasks that determine the sub-space of possible solutions [1]. Once the model and the governing equations have been selected, the method of solution can also influence how realistic the end result will be. In the traditional earthquake engineering studies of response, structures have been represented both by lumped mass and by continuous models, but the prevailing methods of the solution have usually followed the vibrational approach, using the superposition of modal responses.

The vibrational approach for the solution of the response of structures, which are excited at their base by earthquake shaking, was formulated by Biot in early 1930s [2-4]. In this approach, the linear response is represented by superposition of the responses of the equivalent degrees of freedom, corresponding to the responses for the

\footnotetext{
${ }^{*}$ Corresponding author. Tel.: + 12137400570 .

E-mail address: trifunac@usc.edu (M.D. Trifunac).
}

generalized coordinates, whose amplitudes in time, determined by excitation, are expressed via the Duhamel's integral [5]. It can be shown that for the linear systems such a representation is complete.

In engineering applications, the vibrational representation of the solution is further simplified by considering only the fundamental and, occasionally, a few of the lowest frequencies of the system. Doing so is analogous to lowpass filtering of the complete solution [5,6], but it can work well when the excitation is small. However, in the near field of earthquakes, the ground motion may contain large and short displacement pulses, the duration of which can be shorter than the fundamental period of the structure. For this type of excitation, the response spectrum superposition method ceases to be valid and must be replaced by a solution in terms of propagating waves. As will be shown in this paper, for short impulsive ground motions the damage can occur before the wave entering the structure completes its travel up and down the structure, and well before the wave interference can occur - that is, well before the physical conditions can lead to the interference of waves and creation of the mode shapes.

Studies of the response using the wave propagation approach are not new, but the method of analysis has only 
recently started to be used for interpretation of the recorded response, for modeling a two-dimensional response, and for interpretation of full-scale experimental measurements of structural vibrations. Wave propagation models of buildings have been used for many years [7] but are only recently beginning to be verified against observations [8-12]. Continuous, 2-dimensional wave propagation models (homogeneous, horizontally layered and vertically layered, shear plates) can be employed to study the effects of traveling waves on the response of long buildings [13-18]. Discrete-time, 1-dimensional wave propagation models were proposed to study the response of tall buildings [19], and 2-dimensional finite difference methods were used to study linear and non-linear soil-structure interaction [20].

In this paper, because of the one-dimensional nature of the model adopted for the analysis, the two- and threedimensional effects of soil-structure interaction will be ignored [21,22], and thus significant mechanisms of energy loss (non-linear response of the soil and radiation damping) will be neglected. Other simplifications in this paper are that the dynamic instability and the effects of gravity on non-linear response will not be considered [22-25].

In this paper, we will describe the most elementary form of the 1-dimensional shear wave propagation in the structure with elasto-plastic material properties. We will use finite differences to calibrate the response, and we will focus mainly on the most elementary aspects and consequences of non-linear response. After a brief presentation on the method of computation and a description of the transparent boundary conditions in the soil, we will describe the results of 1-dimensional waves in an elastoplastic continuum subjected to shear pulses of strong motion.

The work presented in this paper should also contribute to a better understanding of how and where the incident wave energy is distributed and dissipated within the structure and in the structure-soil system. This understanding is essential for further development of the power design method [6].

\section{Model and numerical scheme}

We consider horizontal deformations, $u$, in a onedimensional shear beam supported by one-dimensional half space and excited by a vertically propagating shear wave described by a half-sine-pulse (Fig. 1). As noted by Dablain [26], a finite difference scheme for a solution of this problem with accuracy $\mathrm{O}\left(\Delta t^{2}, \Delta x^{2}\right)$, where $\Delta x$ and $\Delta t$ are the space and time increments, leads to the exact solution for $\frac{\beta \Delta t}{\Delta x}=1$, where $\beta$ is the velocity of shear waves. With a ratio of the spatial intervals $\frac{\Delta x_{\mathrm{b}}}{\Delta x_{\mathrm{s}}}=\frac{\beta_{\mathrm{b}}}{\beta_{\mathrm{s}}}$ we can meet this requirement. For simplicity, the incident displacement in the soil is chosen to be a sinusoidal pulse with characteristics shown in Fig. 1.

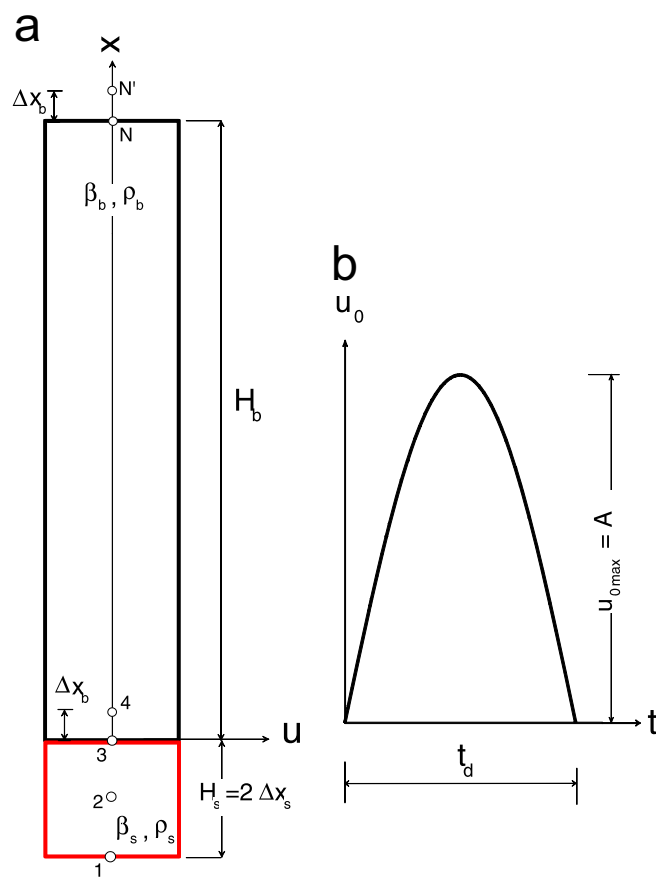

Fig. 1. Shear beam and incoming strong-motion displacement pulse: (a) model of the beam, and (b) the pulse in the soil.

The equation of motion is

$v_{t}=\frac{1}{\rho}(\sigma)_{x}$,

and the relation between the derivative of the strain and the velocity is

$\varepsilon_{t}=v_{x}$,

where $v, \rho, \sigma$, and $\varepsilon$ are particle velocity, density, shear stress, and shear strain, and the subscripts $t$ and $x$ represent derivatives with respect to time and space.

The domain consists of two materials (see Fig. 1): (1) $-2 \Delta x_{\mathrm{s}} \leqslant x<0$ with physical properties $\rho_{\mathrm{s}}$ and $\mu_{\mathrm{s}}$, and (2) $0<x \leqslant H_{\mathrm{b}}$ with physical properties $\rho_{\mathrm{b}}$ and $\mu_{\mathrm{b}}$-for linear response, where $\rho_{\mathrm{i}}$ is the density and $\mu_{i}$ is the shear modulus in the soil $(i=\mathrm{s})$ or in the building $(i=\mathrm{b}) \cdot v=\frac{\partial u}{\partial t}$ and $\varepsilon=\frac{\partial u}{\partial x}$ are the velocity and the strain of a particle, and $u$ is out-ofplane displacement of a particle perpendicular to the propagation ray.

It is assumed that the incoming wave is known and that its displacement as a function of $t$ is prescribed at the point $1\left(x=-2 \Delta x_{\mathrm{s}}\right)$. Also, in this paper it is assumed that the soil is always in the linear elastic state. The Lax-Wendroff finite difference method [27] for a set of simultaneous equations is used to solve the problem.

A mesh with different spatial intervals in the soil and in the building will be used. The spatial intervals are defined by

$\Delta x_{i}=\beta_{i} \cdot \Delta t$,

where $\beta_{i}$ is the velocity of shear waves in the soil $(i=\mathrm{s})$ or in the building $(i=\mathrm{b})$ and $\Delta t$ is the time step. 
The building is discretized into 197 spatial intervals and the soil into 2, so the properties of the mesh are

$\Delta x_{\mathrm{b}}=\frac{H_{\mathrm{b}}}{197} \quad$ and $\quad \Delta x_{\mathrm{s}}=\frac{\beta_{\mathrm{s}}}{\beta_{\mathrm{b}}} \Delta x_{\mathrm{b}}$.

The cell length at the interface point $3(x=0)$ is

$\Delta x_{3}=\frac{\left(\Delta x_{\mathrm{b}}+\Delta x_{\mathrm{s}}\right)}{2}$,

and the initial equivalent shear modulus for this point is obtained from the condition of continuity of the displacements and the stresses at the interface (see Appendix A) as

$\mu_{3}=\frac{\mu_{\mathrm{s}} \mu_{\mathrm{b}}\left(\Delta x_{\mathrm{s}}+\Delta x_{\mathrm{b}}\right)}{\mu_{\mathrm{s}} \Delta x_{\mathrm{b}}+\mu_{\mathrm{b}} \Delta x_{\mathrm{s}}}$.

The yielding strain in the building is $\varepsilon_{y b}$, and to maintain the continuity of the stresses at the interface the yielding strain in contact point 3 is obtained from $\mu_{\mathrm{b}} \varepsilon_{\mathrm{yb}}=\mu_{3} \varepsilon_{\mathrm{y} 3}$ (Fig. 2)

$\varepsilon_{\mathrm{y} 3}=\frac{\mu_{\mathrm{b}}}{\mu_{3}} \cdot \varepsilon_{\mathrm{yb}}$,

where $\mu_{\mathrm{b}}$ is the shear modulus in the building and $\mu_{3}$ is the equivalent shear modulus at the contact point 3 . The time step is constant during the analysis, as follows:

$\Delta t=\frac{\Delta x_{\mathrm{b}}}{\beta_{\mathrm{b}}}=\frac{\Delta x_{\mathrm{s}}}{\beta_{\mathrm{s}}}$.

Above the top point $N$, an additional dummy point $N^{\prime}$ is introduced at distance $\Delta x_{\mathrm{b}}$. For a stress-free point $N$, for all time, the velocities and the stress at the point $N^{\prime}$ are updated as

$v_{N^{\prime}}=v_{N-1}$,

$\sigma_{N^{\prime}}=-\sigma_{N-1}$.

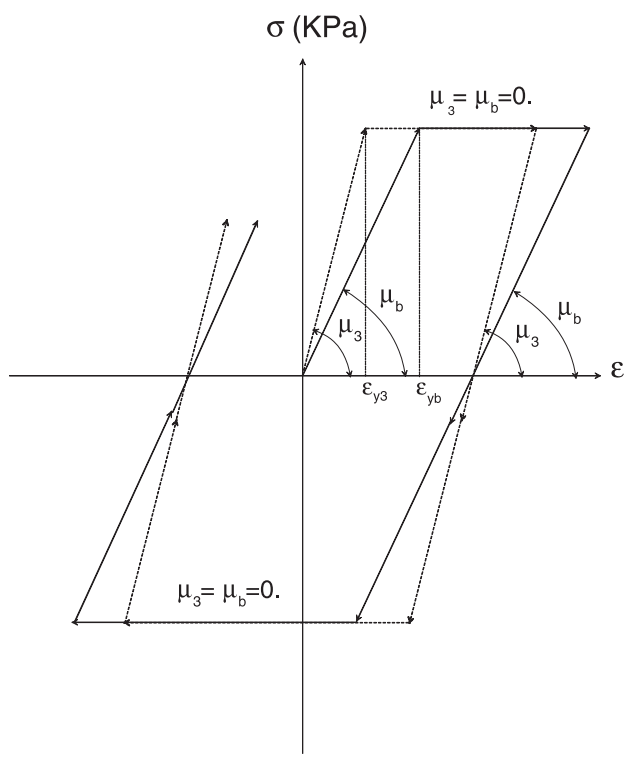

Fig. 2. The constitutive laws, $\sigma-\varepsilon$, for the building (solid line) and for the interface (dashed line).
Eqs. (2.1) can be written in vector form as

$\frac{\partial \mathbf{U}}{\partial t}=\frac{\partial \mathbf{F}}{\partial x}$

where

$\mathbf{U}=\left\{\begin{array}{l}v \\ \varepsilon\end{array}\right\} \quad$ and $\quad \mathbf{F}=\left\{\begin{array}{c}\sigma \\ \rho \\ v\end{array}\right\}=\left\{\begin{array}{c}\frac{\mu(\varepsilon) \cdot \varepsilon}{\rho} \\ v\end{array}\right\}$.

The vector $\mathbf{U}$ at point $i$ in time $(j+1) \Delta t$ expanded in Taylor series is

$$
\begin{aligned}
\mathbf{U}_{i, j+1}= & \mathbf{U}_{i, j}+\Delta t\left(\frac{\partial \mathbf{U}}{\partial t}\right)_{i, j}+\frac{\Delta t^{2}}{2}\left(\frac{\partial^{2} \mathbf{U}}{\partial t^{2}}\right)_{i, j} \\
& +\mathrm{O}\left(\Delta t^{3}\right), \text { and from Eq. (2.7), } \\
\mathbf{U}_{i, j+1}= & \mathbf{U}_{i, j}+\Delta t\left(\frac{\partial \mathbf{F}}{\partial x}\right)_{i, j}+\frac{\Delta t^{2}}{2} \frac{\partial}{\partial t}\left(\frac{\partial \mathbf{F}}{\partial x}\right)_{i, j}+\mathrm{O}\left(\Delta t^{3}\right), \\
\mathbf{U}_{i, j+1}= & \mathbf{U}_{i, j}+\Delta t\left(\frac{\partial \mathbf{F}}{\partial x}\right)_{i, j}+\frac{\Delta t^{2}}{2} \frac{\partial}{\partial x}\left(\mathbf{A}(\mathbf{U}) \frac{\partial \mathbf{F}}{\partial x}\right)_{i, j}+\mathrm{O}\left(\Delta t^{3}\right) .
\end{aligned}
$$

$\mathbf{A}(\mathbf{U})$ is the Jacobian matrix

$\mathbf{A}(\mathbf{U})=\frac{\partial \mathbf{F}}{\partial \mathbf{U}}=\left[\begin{array}{ll}\frac{\partial \sigma}{\rho \partial v} & \frac{\partial \sigma}{\rho \partial \varepsilon} \\ \frac{\partial v}{\partial v} & \frac{\partial v}{\partial \varepsilon}\end{array}\right]=\left[\begin{array}{cc}0 & \frac{1}{\rho} \frac{\mathrm{d} \sigma}{\mathrm{d} \varepsilon} \\ 1 & 0\end{array}\right]$.

\section{Transparent boundary}

The transparent boundary adopted for this study is described in Fujino and Hakuno [28] (Fig. 3). This is a perfect transparent boundary for one-dimensional waves, when $\frac{\beta \Delta t}{\Delta x}=1$. In Fig. 3, the horizontal axis is time and the vertical axis is space. The points are represented by their discrete coordinates. The first coordinate represents discrete space, while the second coordinate represents discrete time, so the column consisting of spatial points 1-3 represents a certain time step.

Point 1 is where the prescribed displacement is applied. We assume that this displacement travels upward in each time step. Point 2 is the boundary point of the model, where the quantities of motion are updated in each time step. Point 3 is the first spatial point, where the motion is computed using finite differences.

The motion at each point results from two components of motion, one from a wave going up and one from a wave going down. To update the motion at boundary point 2 , in time step $k$, we proceed as follows. The total motion at 2 is

$u(2, k)=\uparrow u(2, k)+\downarrow u(2, k)$,

where the arrows denote the direction of wave propagation ( $\uparrow$ for up and $\downarrow$ for down). The motion at point 1 results from up-going wave $u(1, t / \Delta t)=\uparrow u(1, t / \Delta t)=u_{0}(t / \Delta t)$. 


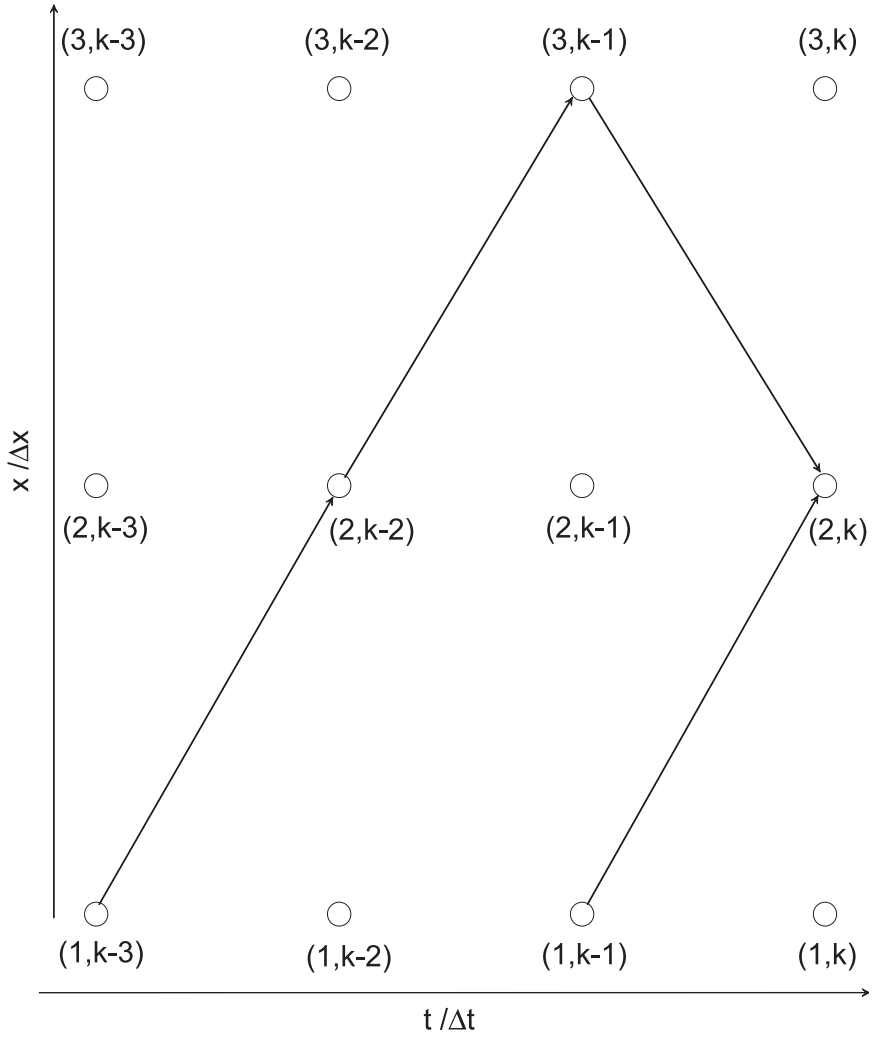

Fig. 3. The model of the absorbing boundary.

Then,

$\uparrow u(2, k)=u(1, k-1)=u_{0}(k-1)$.

The component of motion from the wave traveling down is

$\downarrow u(2, k)=\downarrow u(3, k-1)$.

From $u(3, k-1)=\uparrow u(3, k-1)+\downarrow u(3, k-1)$ it follows that

$\downarrow u(3, k-1)=u(3, k-1)-\uparrow u(3, k-1)$.

The motion at point 3 at time step $(k-1)$ from the wave traveling upward is the motion at point 2 from the wave traveling upward in the previous time step $(k-2)$. From Eq. (3.2), the motion at point 2 from the wave traveling upward in time step $(k-2)$ is the given motion at time step $(k-3)$, so that when we have $\uparrow u(3, k-1)=\uparrow$ $u(2, k-2)=u_{0}(k-3)$.

Eq. (3.3) becomes

$\downarrow u(2, k)=\downarrow u(3, k-1)=u(3, k-1)-u_{0}(k-3)$.

Substituting Eq. (3.2) and Eq. (3.5) into Eq. (3.1), the motion at point 2 is

$u(2, k)=u_{0}(k-1)+u(3, k-1)-u_{0}(k-3) \quad \forall k$.

Eq. (3.6) is the boundary condition at the transparent boundary point 2 , where $u$ stands for displacement, velocity, strain, or stress.

For the linear case at the contact (point 3), one part of the incoming wave is transmitted into the other medium and one is reflected back into the same medium. The corresponding coefficients are obtained from the boundary conditions of continuity of the displacements and stresses at the contact. For a transmitted wave from medium B to medium $\mathrm{A}$, and for a reflected wave from medium $\mathrm{B}$ back into medium $\mathrm{B}$, the above coefficients are

$$
k_{\mathrm{trB} \rightarrow \mathrm{A}}=\frac{2}{1+\frac{\frac{\mu_{\mathrm{a}}}{\mu_{\mathrm{b}}}}{\beta_{\mathrm{b}}}}=\frac{2}{1+\frac{\rho_{\mathrm{a}} \beta_{\mathrm{a}}}{\rho_{\mathrm{b}} \beta_{\mathrm{b}}}} .
$$

And, similarly,

$k_{\mathrm{refB} \rightarrow \mathrm{B}}=\frac{1-\frac{\rho_{\mathrm{a}} \beta_{\mathrm{a}}}{\rho_{\mathrm{b}} \beta_{\mathrm{b}}}}{1+\frac{\rho_{\mathrm{a}} \beta_{\mathrm{a}}}{\rho_{\mathrm{b}} \beta_{\mathrm{b}}}}$.

For the opposite direction of propagation, the numerators and the denominators in these fractions exchange places.

\section{Numerical examples}

For the numerical examples, we consider a shear beam supported by elastic soil, as shown in Fig. 1. The densities of the soil and of the beam are assumed to be the same: $\rho_{\mathrm{b}}=\rho_{\mathrm{s}}=\rho=2000 \mathrm{~kg} / \mathrm{m}^{3}$. The velocity of the shear waves in the soil is taken as $\beta_{\mathrm{s}}=250 \mathrm{~m} / \mathrm{s}$, and in the building as $\beta_{\mathrm{b}}=100 \mathrm{~m} / \mathrm{s}$. To study non-linear response and the development of permanent deformations in the beam, we introduce two dimensionless parameters:

- dimensionless amplitude

$$
\alpha=\frac{A}{H_{\mathrm{b}} \cdot \varepsilon_{\mathrm{yb}}},
$$

where $A$ is the amplitude of the pulse (see Fig. 1), $H_{\mathrm{b}}$ is the height of the building, and $\varepsilon_{\mathrm{yb}}$ is the yielding strain in the building; and

- dimensionless frequency

$$
\eta=\frac{H_{\mathrm{b}}}{\frac{\lambda_{\mathrm{b}}}{2}}=\frac{H_{\mathrm{b}}}{\frac{\beta_{\mathrm{b}} \cdot 2 t_{\mathrm{d}}}{2}}=\frac{H_{\mathrm{b}}}{\beta_{\mathrm{b}} t_{\mathrm{d}}},
$$

where $\lambda_{\mathrm{b}}$ is the wavelength of the wave in the building, $\beta_{\mathrm{b}}$ is the shear wave velocity in the building, and $t_{\mathrm{d}}$ is the duration of the half-sine pulse.

To understand the development of the permanent strain in the non-linear beam, we analyze first the solution for the linear beam. 
The displacement and the strain for the linear beam are

$$
u(x, t)=A \sum_{j=1}^{\infty} k_{j}\left\{\begin{array}{l}
\sin \frac{\pi}{t_{\mathrm{d}}}\left(t-t_{j-1}-\frac{x}{\beta_{\mathrm{b}}}\right)\left[H\left(t-t_{j-1}-\frac{x}{\beta_{\mathrm{b}}}\right)\right. \\
\left.-H\left(t-t_{j-1}-\frac{x}{\beta_{\mathrm{b}}}-t_{\mathrm{d}}\right)\right] \\
+\sin \frac{\pi}{t_{\mathrm{d}}}\left(t-t_{j}+\frac{x}{\beta_{\mathrm{b}}}\right)\left[H\left(t-t_{j}+\frac{x}{\beta_{\mathrm{b}}}\right)\right. \\
\left.-H\left(t-t_{j}+\frac{x}{\beta_{\mathrm{b}}}-t_{\mathrm{d}}\right)\right]
\end{array}\right\}
$$

and

$$
\begin{aligned}
\varepsilon(x, t)=A \frac{\pi}{\beta_{\mathrm{b}} t_{\mathrm{d}}} \sum_{j=1}^{\infty} k_{j} \\
\quad \times\left\{\begin{array}{l}
-\cos \frac{\pi}{t_{\mathrm{d}}}\left(t-t_{j-1}-\frac{x}{\beta_{\mathrm{b}}}\right)\left[H\left(t-t_{j-1}-\frac{x}{\beta_{\mathrm{b}}}\right)\right. \\
\left.-H\left(t-t_{j-1}-\frac{x}{\beta_{\mathrm{b}}}-t_{\mathrm{d}}\right)\right] \\
+\cos \frac{\pi}{t_{\mathrm{d}}}\left(t-t_{j}+\frac{x}{\beta_{\mathrm{b}}}\right)\left[H\left(t-t_{j}+\frac{x}{\beta_{\mathrm{b}}}\right)\right. \\
\left.-H\left(t-t_{j}+\frac{x}{\beta_{\mathrm{b}}}-t_{\mathrm{d}}\right)\right]
\end{array}\right\},
\end{aligned}
$$

where $j$ is the order number of the passage of the wave on the path bottom-top-bottom in the building, $t_{j}=\frac{2 j H_{\mathrm{b}}}{\beta_{\mathrm{b}}}(j=$ $0,1,2,3, \ldots$,$) is the time required for the wave to pass j$ times the path bottom-top-bottom (two heights),

$k_{j}=k_{t} k_{r}^{j-1}$

is the amplitude factor of the pulse in the soil, in its $j$ th passage along the path bottom-top-bottom, through the building, and $k_{t}$ and $k_{r}$ are coefficients defined by Eqs. (3.7) and (3.8). The amplitudes of $k_{j}=k_{j}(j)$ and $k_{j}=\left|k_{j}(j)\right|$, for the examples considered in this paper, are shown in Fig. 4.

The odd terms in the series of Eqs. (4.3) and (4.4) describe the response at arbitrary points to the pulse coming from below, while the even terms describe the response for the pulse arriving from above. For the shear wave velocities in our example, $\quad\left(\beta_{\mathrm{s}}=250 \mathrm{~m} / \mathrm{s}\right.$ and $\left.\beta_{\mathrm{b}}=100 \mathrm{~m} / \mathrm{s}\right), k_{t}=\frac{10}{7}$, and $k_{r}=-\frac{3}{7}$. In Eq. (4.3) the displacement is positive for odd passages and negative for even passages. The displacement and velocity change sign after reflection from the soil-building interface and do not change sign after reflection from the top of the building. The strain changes sign after reflection from the top of the building and does not change sign after reflection from the building-soil interface.

The constant multiplying the series in Eq. (4.4) in terms of dimensionless amplitude and dimensionless frequency is

$A \frac{\pi}{\beta_{\mathrm{b}} t_{\mathrm{d}}}=A_{\varepsilon}=\pi \alpha \eta \varepsilon_{\mathrm{yb}}$.

To analyze the occurrence of permanent strain, we consider two characteristic points in the building: (1) Point B $(x=0)$ at the soil-building interface (point 3 in the grid, see Fig. 1), and (2) Point $\mathrm{T}\left(x=H_{\mathrm{b}}-\frac{\beta_{\mathrm{b}} \cdot t_{\mathrm{d}}}{2}\right)$, where the amplitudes of the strain with the same sign meet after reflection from the top of the building. The location of this point is dependent upon the duration (wavelength) of the pulse. To find the location of this point and the time of occurrence of the local maximum strain, we use Eq. (4.4).

The first term in Eq. (4.4) is one if the argument of the cosine function is equal to $t_{\mathrm{d}}\left(t-t_{0}-\frac{x}{\beta_{\mathrm{b}}}=t_{\mathrm{d}}\right)$. The second term is one if the argument of the second cosine function is equal to $0\left(t-t_{1}+\frac{x}{\beta_{\mathrm{b}}}=0\right)$.

The position of point $\mathrm{T}$, where the strain amplitude is two times larger than the strain entering the beam, is at $x=H_{\mathrm{b}}-\frac{\beta_{\mathrm{b}} \cdot t_{\mathrm{d}}}{2}$, and the time when this occurs is $t=\frac{H_{\mathrm{b}}}{\beta_{\mathrm{b}}}+\frac{t_{\mathrm{d}}}{2}$. From Eq. (4.4) in the first passage of the pulse, $t<\frac{2 H_{\mathrm{b}}}{\beta_{\mathrm{b}}}$, only the first term in the series exists. The strain at point $\mathrm{B}$ reaches its absolute maximum at the very beginning, during the entrance of the pulse into the building, and its value is

$\left|\varepsilon_{\mathrm{B} \max }^{1}\right|=\pi \alpha \eta \varepsilon_{\mathrm{yb}} k_{t}$.

If the strain in Eq. (4.7) is greater than the yielding strain in the building, a permanent strain at the interface will develop. The condition for occurrence of permanent strain at this point is $\left|\varepsilon_{\mathrm{B} \text { max }}^{1}\right|>\varepsilon_{\mathrm{yb}}$, or, in terms of the dimensionless parameters,

$\alpha \eta>\frac{1}{\pi k_{t}}=\frac{\beta_{\mathrm{b}}+\beta_{\mathrm{s}}}{2 \pi \beta_{\mathrm{s}}}=C_{\mathrm{B}}$.

At point $\mathrm{T}$ (this point does not exist if $t_{\mathrm{d}}>\frac{2 H_{\mathrm{b}}}{\beta_{\mathrm{b}}}$, and it coincides with point $\mathrm{B}$ if $\left.t_{\mathrm{d}}=\frac{2 H_{\mathrm{b}}}{\beta_{\mathrm{b}}}\right)$, from Eq. (4.4), the maximum strain during the first passage occurs at $t=$ $\frac{H_{\mathrm{b}}}{\beta_{\mathrm{b}}}+\frac{t_{\mathrm{d}}}{2}$, and its amplitude is $2 A_{\varepsilon} \cdot k_{t}$. The condition for occurrence of the permanent strain is

$\alpha \eta>\frac{1}{2 \pi k_{t}}=\frac{\beta_{\mathrm{b}}+\beta_{\mathrm{s}}}{4 \pi \beta_{\mathrm{s}}}=C_{\mathrm{T}}=\frac{C_{\mathrm{B}}}{2}$.

For the shear wave velocities in our example $C_{\mathrm{B}}=0.2228$ and $C_{\mathrm{T}}=0.1114$.

When the reflected wave from the top of the building reaches the soil-building interface $\left(t=t_{1}\right)$, the wave begins the second passage. The linear solution for the strain in Eq. (4.4) at B now involves three terms in the series if the duration of the pulse is longer than $\frac{2 H_{\mathrm{b}}}{\beta_{\mathrm{b}}}$, and two terms for shorter pulses. Recalling Eqs. (4.4) and (4.6), the solution at time $t=\frac{2 H_{\mathrm{b}}}{\beta_{\mathrm{b}}}=t_{1}$ is

$\varepsilon\left(0, t_{1}\right)=A_{\varepsilon}\left\{k_{1}\left[-\cos \left(\frac{\pi}{t_{\mathrm{d}}} \cdot \frac{2 H_{\mathrm{b}}}{\beta_{\mathrm{b}}}\right)+\cos 0\right]-k_{2} \cdot \cos 0\right\}$ 

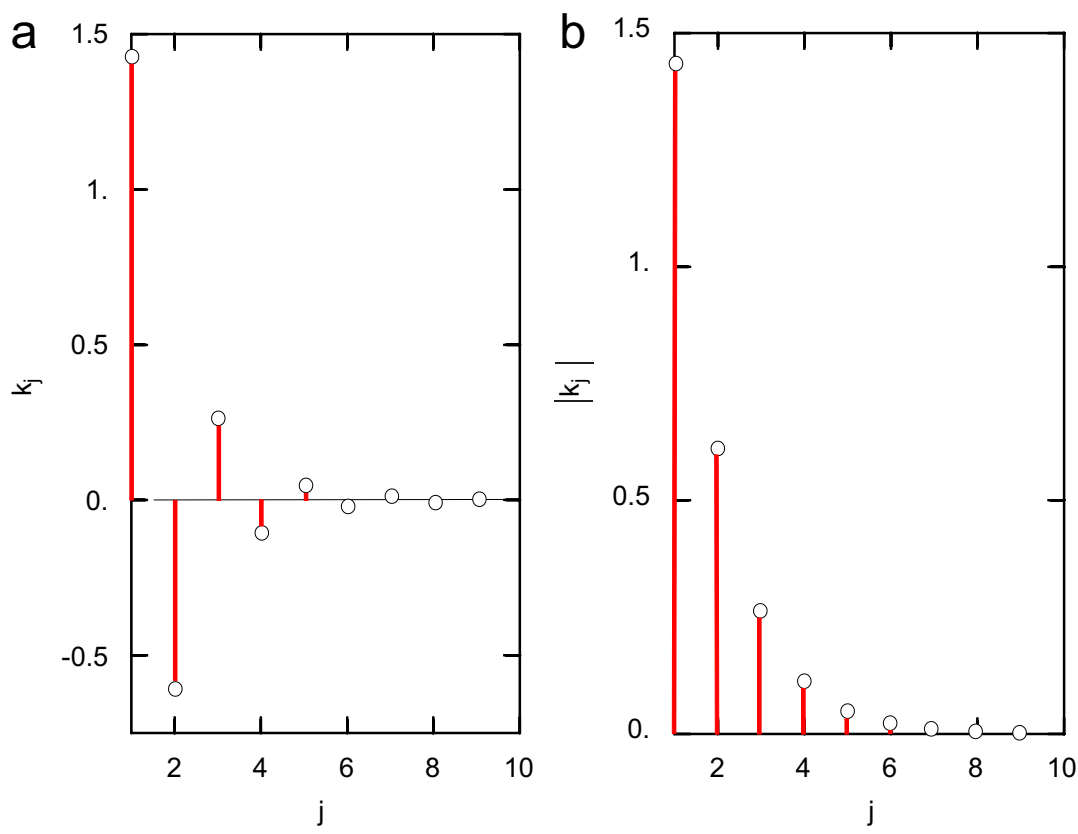

Fig. 4. Dependence of $k_{j}(j)$ on $j$.

or

$\varepsilon\left(0, t_{1}\right)=\pi \alpha \eta \varepsilon_{\mathrm{yb}} k_{t}\left(1-\cos 2 \pi \eta-k_{r}\right)$,

when

$t_{\mathrm{d}}>\frac{2 H_{\mathrm{b}}}{\beta_{\mathrm{b}}} \quad\left(\eta<\frac{1}{2}\right)$,

and

$\varepsilon\left(0, t_{1}\right)=A_{\varepsilon}\left\{k_{1} \cdot \cos 0-k_{2} \cdot \cos 0\right\}$

or

$\varepsilon\left(0, t_{1}\right)=\pi \alpha \eta \varepsilon_{\mathrm{yb}} k_{t}\left(1-k_{r}\right)$,

when

$t_{\mathrm{d}}<\frac{2 H_{\mathrm{b}}}{\beta_{\mathrm{b}}} \quad\left(\eta>\frac{1}{2}\right)$.

Comparing Eqs. (4.7) and (4.10b), because $k_{r}<0$, for shorter pulses $(\eta>0.5)$, the strain at point $\mathrm{B}$, at the beginning of the second passage, is always larger $\left(1+\left|k_{r}\right|\right)$, and for our example, $\frac{10}{7}$ times larger than the strain, $A_{\varepsilon} \cdot k_{t}$, at the beginning of the first passage. However, it is still smaller than the strain at $\mathrm{T}$ in the first passage $\left(2 A_{\varepsilon} \cdot k_{t}\right)$. For shorter pulses $(\eta>0.5)$, it can be concluded that if there is no occurrence of permanent strain during the first passage at point $\mathrm{T}$, the response of the beam will be linear for all time.

For longer pulses $(\eta<0.5)$, comparing Eqs. (4.7) and (4.10a), it can be seen that for $\eta<\frac{1}{2 \pi} \arccos \left(\left|k_{r}\right|\right)$ the strain at interface point $\mathrm{B}$ at the beginning of the second passage is smaller than the strain at the beginning of the first passage, and for $\eta>\frac{1}{2 \pi} \arccos \left(\left|k_{r}\right|\right)$ the former strain is larger than the latter one. For our example $\left(k_{r}=-\frac{3}{7}\right)$, $\eta>0.18$ always gives larger strain at the interface point at the beginning of the second passage than the strain at the beginning of the first passage.

The largest amplification of the strain at B is for $\eta=0.5$, when, at the beginning of the second passage, the strain is $\left(2+\left|k_{r}\right|\right)$ times larger than the strain during the first passage. For our example, the amplification is $\frac{17}{7}$, which gives larger strain than the strain at point $\mathrm{T}$ during the first passage $\left(2 A_{\varepsilon} \cdot k_{t}\right)$. Therefore, for longer pulses $(\eta \leqslant 0.5)$ the first permanent strain can occur later (see Fig. 5). The conditions in Eq. (4.8) can be written also in the form

$\alpha \eta>\frac{1}{\pi k_{t}\left(2+\left|k_{r}\right|\right)}=\frac{\beta_{\mathrm{b}}+\beta_{\mathrm{s}}}{2 \pi \beta_{\mathrm{s}}\left(2+\left|k_{r}\right|\right)}=\frac{C_{\mathrm{B}}}{2+\left|k_{r}\right|}$.

In general, we distinguish three cases for the duration of the pulse and for the corresponding strains at the bottom of the beam at the end of the $j$ th passage $\left(t_{j}\right)$, as follows:

1. $t_{0}<t_{\mathrm{d}}<t_{1}$. The dimensionless frequency is higher than 0.5 .

$\varepsilon\left(0, t_{j}\right)=A_{\varepsilon}\left(k_{j}-k_{j+1}\right)$.

2. $t_{l}<t_{\mathrm{d}}<t_{l+1}, \quad l<j, \quad l \geqslant 1$. The dimensionless frequency is lower than 0.5 , and the pulse has entered into the beam completely. The strain contains $2(l+1)$ terms:

$$
\begin{aligned}
\varepsilon\left(0, t_{j}\right)= & A_{\varepsilon}\left[\sum_{m=j-l}^{j} k_{m} \cos 2 \pi(j-m) \eta\right. \\
& \left.-\sum_{m=j-l+1}^{j+1} k_{m} \cos 2 \pi(j-m+1) \eta\right] .
\end{aligned}
$$



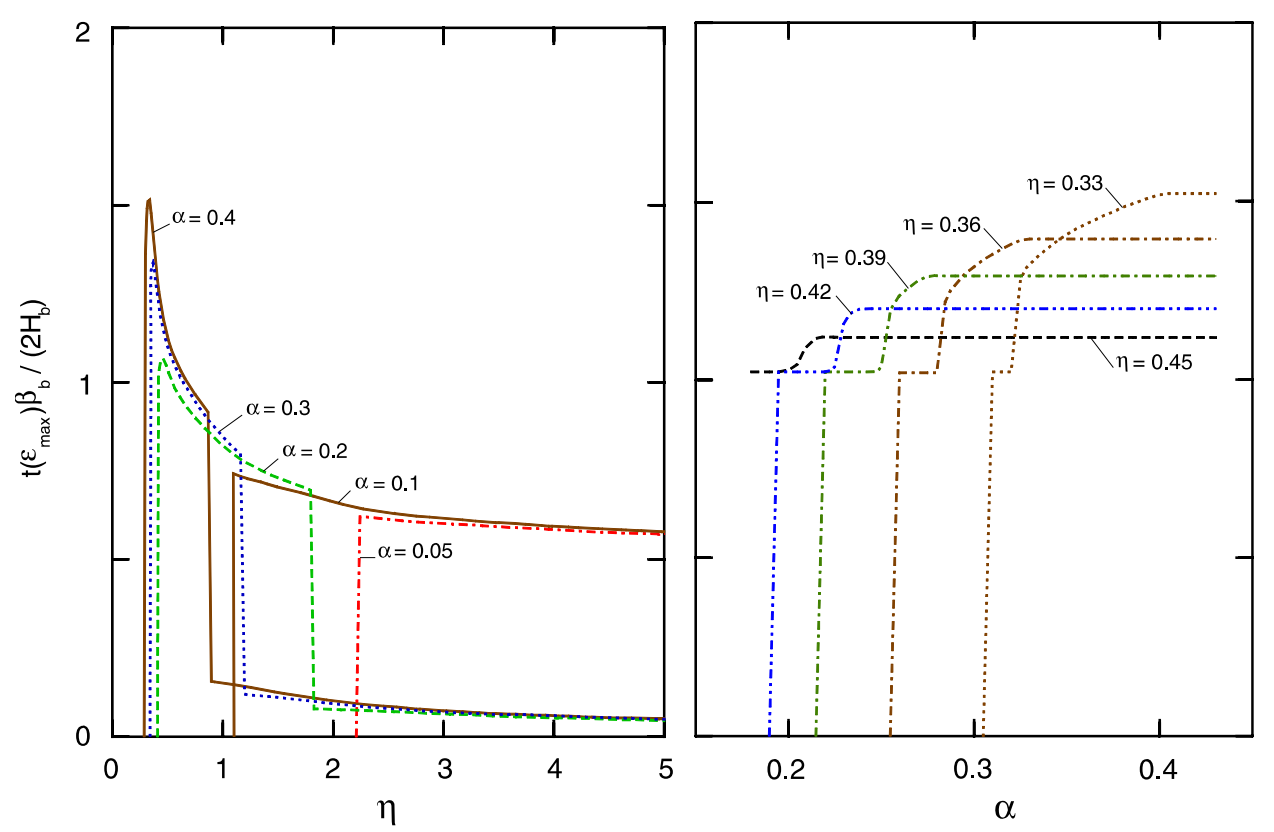

Fig. 5. Normalized time of occurrence of the maximum strain versus $\eta$ - dimensionless frequency (left), and $\alpha$ - dimensionless amplitude (right).

3. $t_{\mathrm{d}}>t_{j}$. The pulse is still entering into the beam. The strain contains $2 j+1$ terms:

$$
\begin{aligned}
\varepsilon\left(0, t_{j}\right)= & -A_{\varepsilon}\left\{\sum_{m=0}^{j} k_{m+1} \cos 2 \pi(j-m) \eta\right. \\
& \left.-\sum_{m=1}^{j} k_{m} \cos 2 \pi(j-m) \eta\right\} .
\end{aligned}
$$

For the non-linear case, we will consider five examples of the dimensionless amplitude $\alpha=0.05 ; 0.1 ; 0.2 ; 0.3$; and 0.4 , in the range of the dimensionless frequency $0.03 \leqslant \eta \leqslant 5$. We will also consider only "small" deformations, for which $\varepsilon \approx \tan \varepsilon$ (geometric linearity).

In Fig. 6a the largest permanent strain in the beam is shown versus the dimensionless frequency. It can be seen that for $\alpha \geqslant 0.2$ the peaks occur in the range $0.36 \leqslant \eta \leqslant 0.46$ (see the conditions described in Eq. (4.8B2). The peak for $\alpha=0.4$ occurs at $\eta=0.36$, with amplitude $\varepsilon_{\max }=0.795$, and it is the largest permanent strain for the range of dimensionless frequencies considered here. The development of the largest permanent strain is explained further in Appendix B. As $\alpha$ decreases, the peak decreases and is shifted toward larger $\eta$ (for $\alpha=0.2$ the peak is at $\eta=0.46$ ). For $\alpha=0.1$ and $\alpha=0.05$ the beam remains linear in this frequency range. Fig. $6 \mathrm{~b}$ shows $\varepsilon_{\max }$ versus $\eta$ for $0.15<\alpha<0.25$ and further illustrates the emergence of the peak at $\eta \sim 0.5$.

As shown above, the coordinate of point $\mathrm{T}$ is dependent upon the duration of the pulse $\left(x_{\mathrm{T}}=H_{\mathrm{b}}-\frac{\beta_{\mathrm{b}} \cdot t_{\mathrm{d}}}{2}\right)$. In terms of the dimensionless frequency and normalizing by the height of the building, $H_{\mathrm{b}}$, the coordinate of the point $\mathrm{T}$ is

$\xi=\frac{x_{\mathrm{T}}}{H_{\mathrm{b}}}=1-\frac{1}{2 \eta}$.

The minimum value of $\eta$ for which point $\mathrm{T}$ is a real (physical) point of the building is $\eta=0.5$, and the maximum value of the dimensionless amplitude for which the building will remain linear for this frequency is $\alpha=$ $\frac{C_{\mathrm{T}}}{\eta}=\frac{0.1114}{0.5}=0.2228$ (see Eq. (4.8T)).

In our example, for $\alpha=0.1$, the first permanent strain will occur at $\mathrm{T}$ for $\eta=1.114$, and for $\alpha=0.05$, at $\mathrm{T}$, for $\eta=2.228$ (see Fig. 6a). As $\eta$ increases, the peak values of $\varepsilon_{\max }$ asymptotically approach constant levels $\varepsilon(\alpha)$. This is suggested by the leveling off of $\varepsilon_{\max }(\eta)$ at large $\eta$.

As can be seen in Fig. 6a, for $\alpha=0.2$, the curve $\varepsilon_{\max }(\eta)$ has a local minimum near $\eta=3$ and a local maximum near $\eta \sim 2.5$. This is further illustrated in Fig. $6 \mathrm{~b}$, where $\varepsilon_{\max }(\eta)$ is plotted for dimensionless amplitudes $\alpha=0.15 ; 0.175 ; 0.2$; 0.225 ; and 0.25 . With $\alpha$ increasing, the local minima and maxima are shifted to the left. Fig. 6a shows that for $\alpha \sim 0.3$ the minimum disappears, and for $\alpha=0.1$ it is near $\eta=5$. This departure from monotonically increasing values appears to be associated with the second natural frequency of the beam on a fixed base.

In Fig. 7a and $\mathrm{b}$ normalized lengths of the segments of the permanently deformed beam are shown versus $\eta$. We distinguish three zones of the beam:

- bottom (deformed) zone,

- middle zone consisting of the lower, not deformed part and the upper deformed part, and

- top (not deformed) zone. 

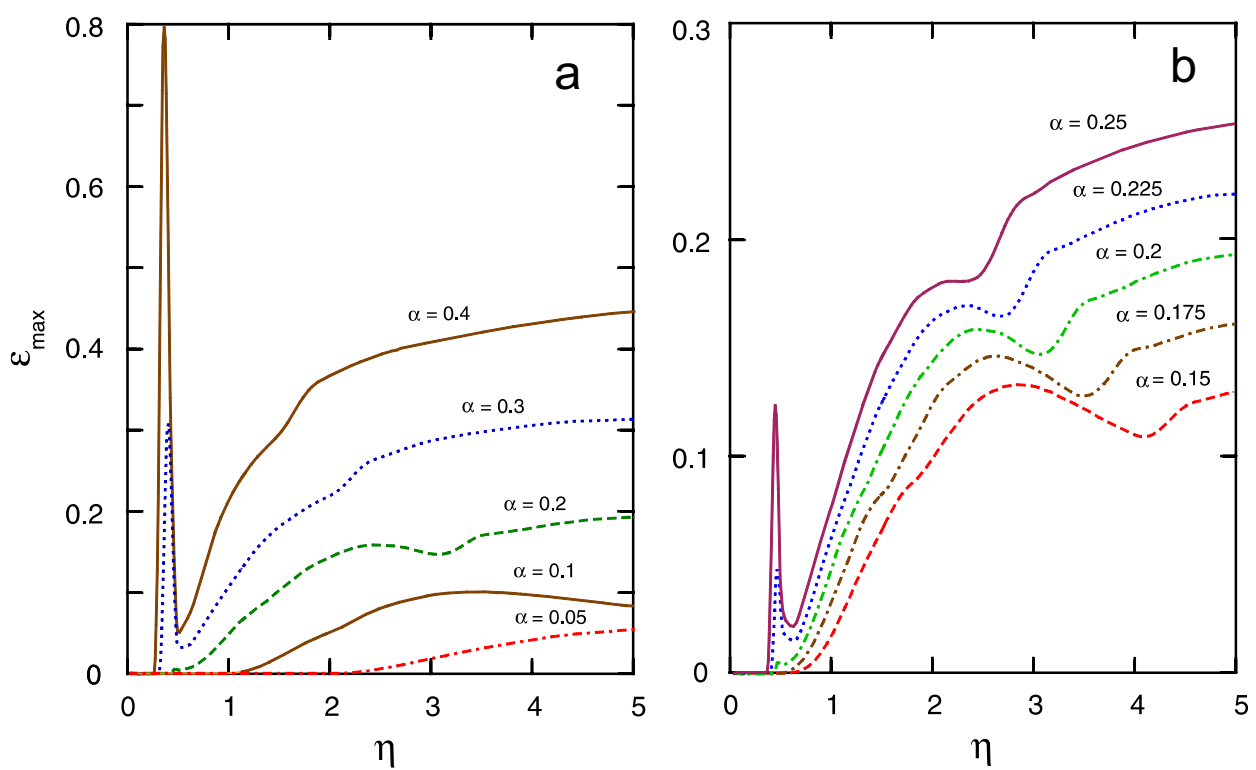

Fig. 6. Maximum permanent strain versus dimensionless frequency $\eta$.

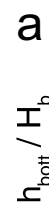

$\underbrace{a}$
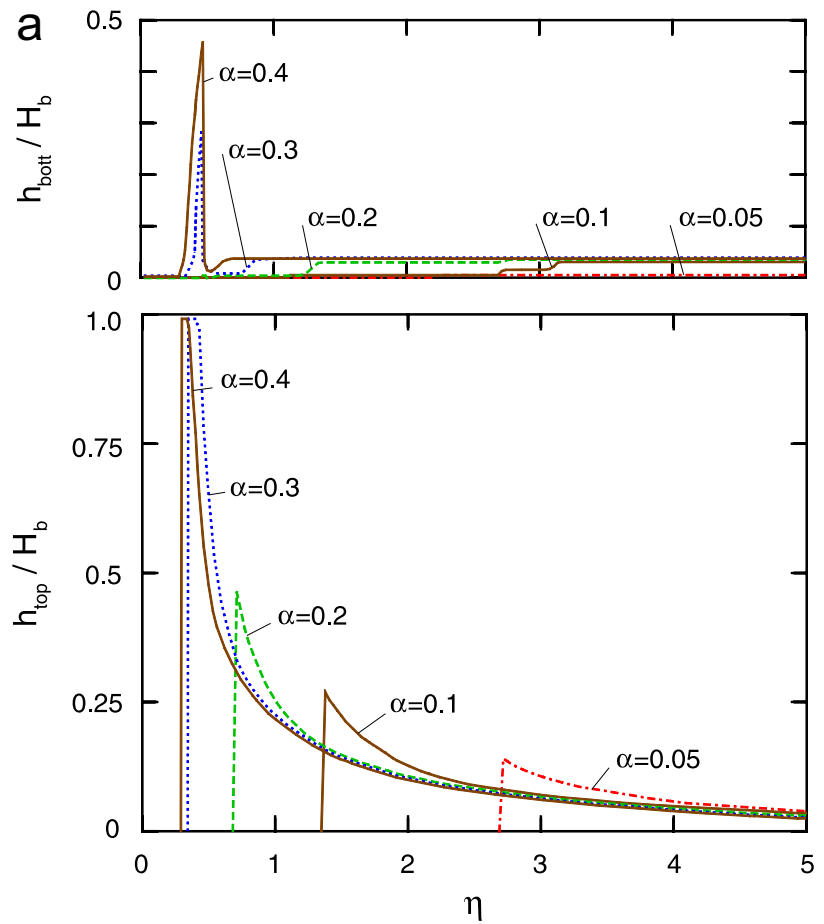

b
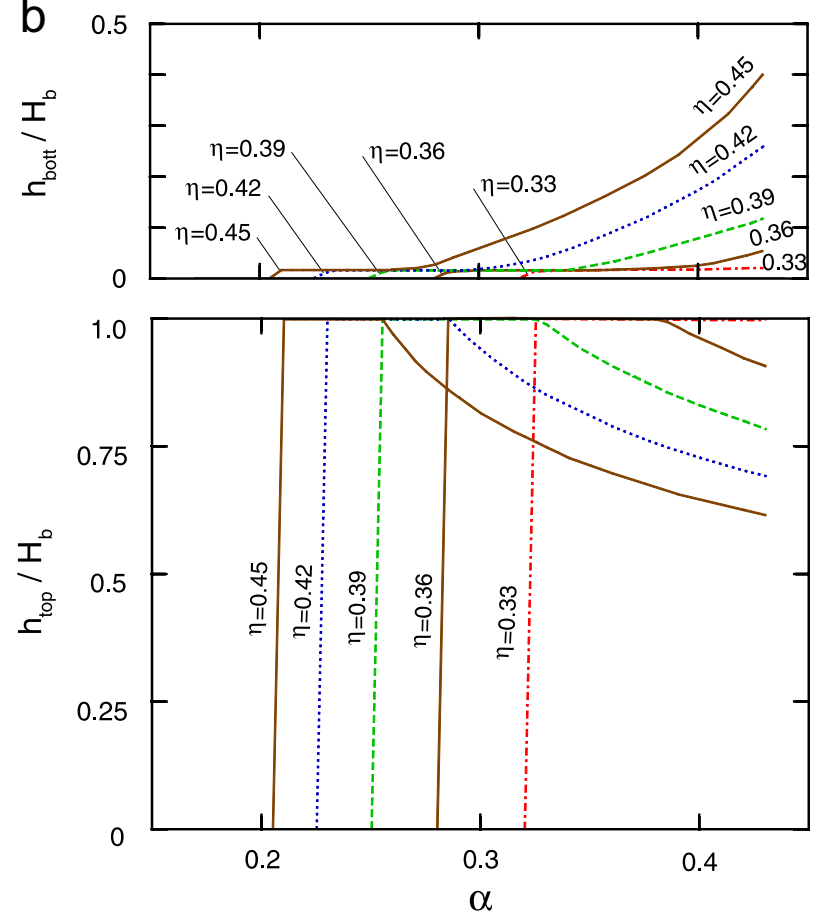

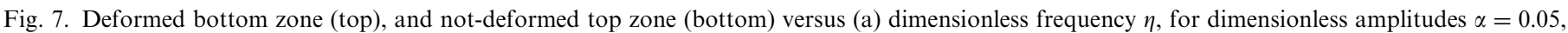
$0.1,0.2,0.3$, and 0.4 ; (b) dimensionless amplitude $\alpha$, for five values of $\eta=0.33,0.36,0.39,0.42$, and 0.45 .

Fig. 7a (top) shows the length of the bottom (deformed) zone, $h_{\text {bott }}$, normalized by the height of the beam, $H_{\mathrm{b}}$. Fig. 7 a (bottom) shows the length of the top (not deformed) zone. In this paper, the numerical criterion adopted for defining these lengths is that if the permanent strain at a point of the building is smaller than $\varepsilon_{\mathrm{yb}} / 5$ the building is treated as being linear at that point. The difference between one, and the sum of the normalized two lengths, $h_{\mathrm{bott}} / H_{\mathrm{b}}$ and $h_{\mathrm{top}} / H_{\mathrm{b}}$ is the normalized length of the middle zone of the beam.
From Fig. 7a it can be seen that for small $\eta$, until the condition in Eq. (4.8B2) is satisfied, the deformed bottom length of the beam is 0 . For the largest amplitude we consider, $\alpha=0.4$, and for, $\eta>\frac{7 C_{B}}{17 \alpha}=0.23$ the condition in Eq. (4.8B2) is satisfied and the permanently deformed zone at the bottom appears. As $\eta$ increases, the length of this zone increases, reaching its maximum value of about $0.46 H_{\mathrm{b}}$ at $\eta=0.5$ (Fig. 7a (top)). After this point, the permanent strain first occurs at point $\mathrm{T}$ (see Eq. (4.8T)), the length of the deformed bottom zone decreases, and the 
middle deformed zone is formed. When the condition in Eq. (4.8B) is satisfied, the length of the deformed bottom part of the beam increases slightly but remains independent on $\eta$, while the top (non-deformed) part decreases. The middle zone consists of a top zone, which is deformed, narrower, and closer to the top as $\eta$ increases, and a bottom linear zone, which lengthens as $\eta$ increases. The situation is similar for $\alpha=0.3$. The maximum length of the deformed zone at the bottom, for this amplitude, is $0.28 H_{\mathrm{b}}$ (see Fig. 7a (top)).

To explain these trends, we recall the analysis of the strains for the linear case. We noted by comparing Eqs. (4.7) and (4.10) that the strain at the beginning of the second passage is larger than at the beginning of the first passage. For long pulses, this can be generalized to the third, fourth, etc., passages of the wave along the path bottom-top-bottom of the beam, if the pulse is long enough so that the first term (which is multiplied with the largest, $k_{1}$ ), still exists in Eq. (4.4). This increase in the strain at the bottom due to multiple reflections increases the length of the deformed zone at the bottom of the building. As the pulse becomes shorter, the permanent strain occurs in the middle or at the top of the building, the wave loses energy to produce these additional permanent strains, and the higher-order passages of the wave are not able to increase the strain at the bottom and the length of the bottom deformed zone. For those conditions, the
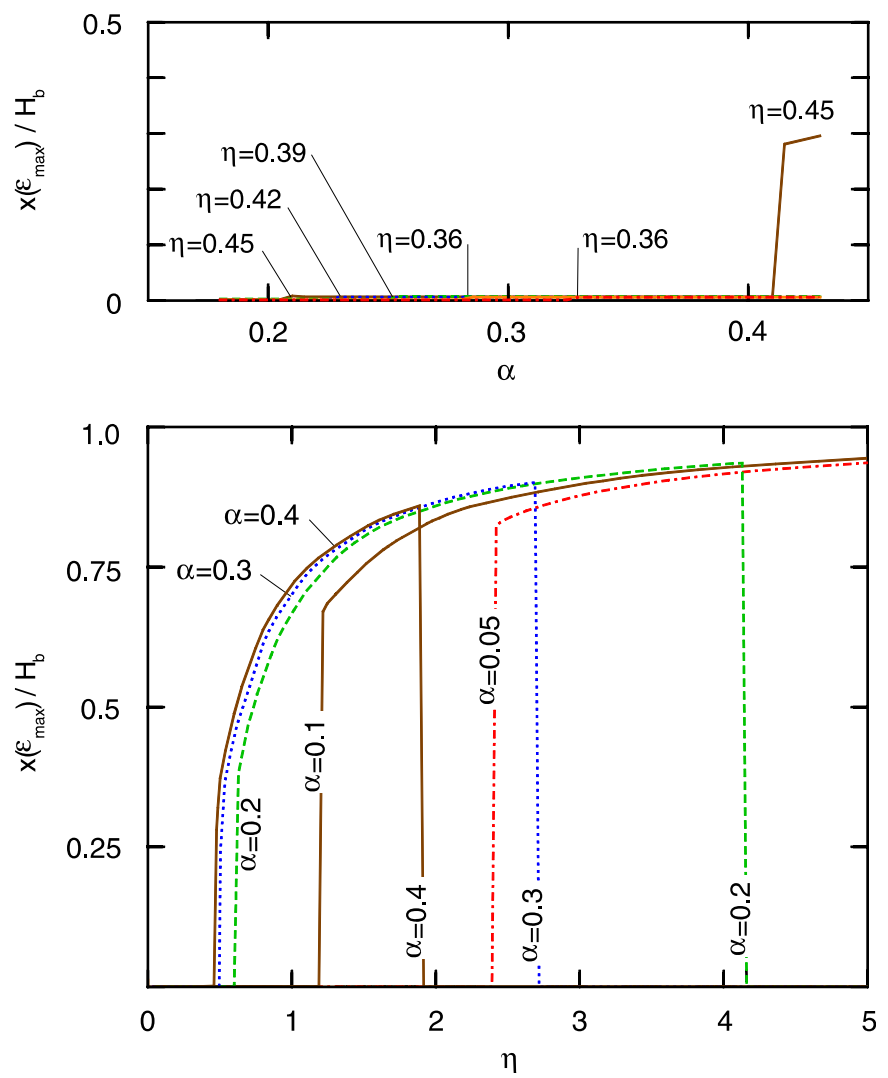

Fig. 8. Location of the point with maximum permanent strain versus dimensionless amplitude $\alpha$ (top), and versus dimensionless frequency $\eta$ (bottom). deformed zone at the bottom is completely formed with the first passage of the wave. This is why there is a sharp decrease in the length of the deformed bottom zone at $\eta=$ 0.5 for $\alpha=0.4$ and 0.3 in our examples. Because this length is completely formed with the first passage of the wave, it stays constant with increasing $\eta$. For smaller $\alpha$ (e.g., $\alpha=0.1$ and 0.05$)$, the condition in Eq. (4.8B2) is not satisfied for long pulses $\eta \leqslant 0.5$, and there is no deformed bottom zone in this frequency range. When the condition in Eq. (4.8T) is satisfied, the beam has a top linear zone, a middle zone with an upper deformed part, and a linear lower part. Finally, when the condition in Eq. (4.8B) is satisfied, all zones exist in the beam.

In Fig. 7b (bottom) the length of the non-deformed, top zone of the building is shown. Again, for small $\eta$ and large dimensionless amplitudes $\alpha$, when Eq. (4.8B2) is satisfied, the length of this zone is the largest. Comparing the plots in Fig. 7a and b, it can be concluded that until the sharp decrease of $h_{\text {top }} / H_{\mathrm{b}}$ in Fig. 7a there is no intermediate zone. After this sharp decrease in Fig. 7a, the length of the top zone in Fig. 7a (bottom) starts to decrease gradually with increase of $\eta$, and the length of the intermediate zone increases with increase of $\eta$.

To further illustrate the appearance of the peak of the permanent strain accompanying long pulses and the three zones in the beam, we consider the dimensionless amplitudes $0.18 \leqslant \alpha \leqslant 0.43$ for five dimensionless frequencies $\eta=0.33 ; 0.36 ; 0.39 ; 0.42$; and 0.45 . In Fig. $7 \mathrm{~b}$ the normalized lengths of the bottom and the top deformed zones of the beam are shown versus $\alpha$. It can be seen from Fig. $7 \mathrm{~b}$ (bottom) that the length of the top, not deformed

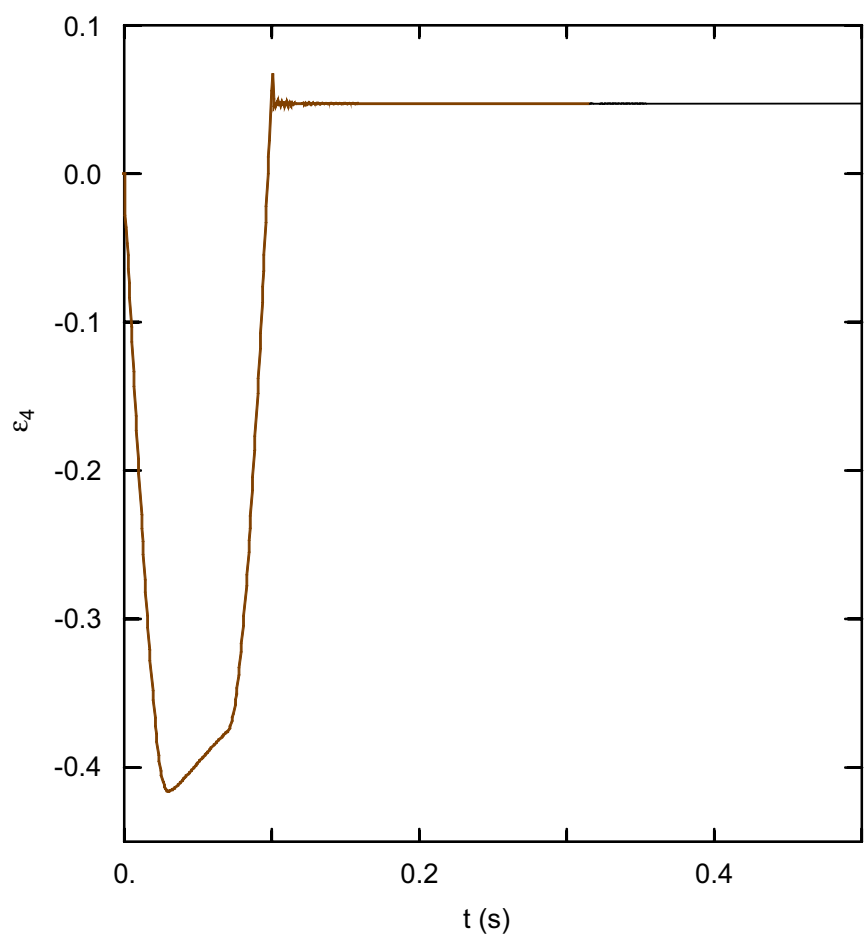

Fig. 9. Strain time history at point $4(x=\Delta x)$, for $\alpha=0.4$ and $\eta=1$. 
zone of the beam starts to decrease as the bottom zone begins to form. We recall the conditions stated in Eqs. (4.8T) and (4.8B2). Let the dimensionless parameters $\alpha$ and $\eta$, at the first appearance of the permanent strain in the building, for long pulses $\eta \leqslant 0.5$ (Eq. (4.8B2)) be $\alpha_{0}$ and $\eta_{0}$. Let these parameters for the appearance of the first permanent strain at T (Eq. (4.8T)) be $\alpha_{1}$ and $\eta_{1}$. Dividing Eq. (4.8B2) by Eq. (4.8T) and using the values for $k_{r}$ adopted for our examples in this paper, we obtain

$\frac{\alpha_{0} \eta_{0}}{\alpha_{1} \eta_{1}}=\frac{14}{17}$.

For the pairs $\left(\eta_{0}, \eta_{1}\right)=(0.39,0.42),(0.36,0.42)$, and $(0.33$, 0.39 ) we obtain $\alpha_{0} / \alpha_{1}=0.95,0.96$, and 0.97 , respectively, or, in general, $\frac{\alpha_{0}}{\alpha_{1}}=\frac{2 \eta_{1}}{\left(2+\left|k_{r}\right|\right) \eta_{0}}$. All of these ratios are close to one, which means that the first permanent strains for $\eta=0.39 ; 0.36 ; 0.33$ in our examples, appear just before the permanent strain occurs in the fictitious point $T$, below point B.

To further investigate where and when the largest strains occur, we turn to Fig. 8. First, we note that for the domain $(\alpha, \eta)$ considered here the condition in Eq. (4.8B) is not satisfied, $\quad \alpha_{\max } \eta_{\max }=0.43 \cdot 0.45=0.1935<C_{\mathrm{B}}=0.2228$, and so there is no permanent strain during the first half of the first passage of the wave through the beam. From Fig. 8 it can be seen that for this range of $\alpha$, except for $\eta=0.45$, the largest permanent strain occurs at the bottom of the beam (point 4 in Fig. 1). For $\eta=0.45$ and for $\alpha \leqslant 0.41$ the largest permanent strain also occurs at the bottom.

The pulse reflected from the top, traveling downward and having positive strain $A_{\varepsilon} k_{t}$ (see Eq. (4.7)), is smaller than the yielding strain $\varepsilon_{\mathrm{yb}}$ (condition in Eq. (4.8T)). It needs some "help" from the part of the pulse traveling upward to reach the yielding strain. For $\eta=0.45$ the duration of the pulse is $t_{\mathrm{d}}=0.2222 \mathrm{~s} ; 0.1111 \mathrm{~s}$ after the first entrance of the pulse, the part of the pulse traveling upward will contribute to the positive strain in the beam. This strain increases with time, having maximum positive value at the end of the pulse. When the reflected pulse meets a large enough supplement of the positive strain from below, it reaches the yielding strain in the beam. With propagation of the reflected pulse and interference with larger strains traveling upward, the lower points of the beam experience permanent strain, as well. In this development of the permanent strain, the pulse reflected from the top loses energy, so the strain at the bottom is weaker than the one with an elastic pulse reflected from the top. For $\alpha>0.41$ and $\eta=0.45$, the strain at $\mathrm{B}$ is smaller than the strain at some point in the beam. For all other parts of the considered domain $(\alpha, \eta)$ the strain at the bottom is the largest.

From Fig. $5 b$ it can be seen that for the range $(\alpha, \eta)$ considered in this paper, the largest strain occurs during the first half of the second passage of the pulse. Until the condition in Eq. (4.8B2) is satisfied for occurrence of the first permanent strain (no permanent strain in the first passage), the time of occurrence of the maximum permanent strain in the beam is the same for all $\eta$. When the permanent strain occurs at some point of the beam during the first passage, the time of maximum strain grows because the maximum strain reflected from the top is smaller than the arriving elastic strain from the half-space propagating upward. The weakening of the reflected strain is greater for larger amplitudes, so that the largest strain at the bottom occurs at a time when the pulse has completely entered the beam $\left(t=t_{\mathrm{d}}\right)$ and the curves in Fig. 5b have constant values $\frac{t_{\mathrm{d}} \beta_{\mathrm{b}}}{2 H_{\mathrm{b}}}=\frac{1}{2 \eta}$. There are transitions (intervals of $\alpha$ ) between these two cases. These transitions are shorter

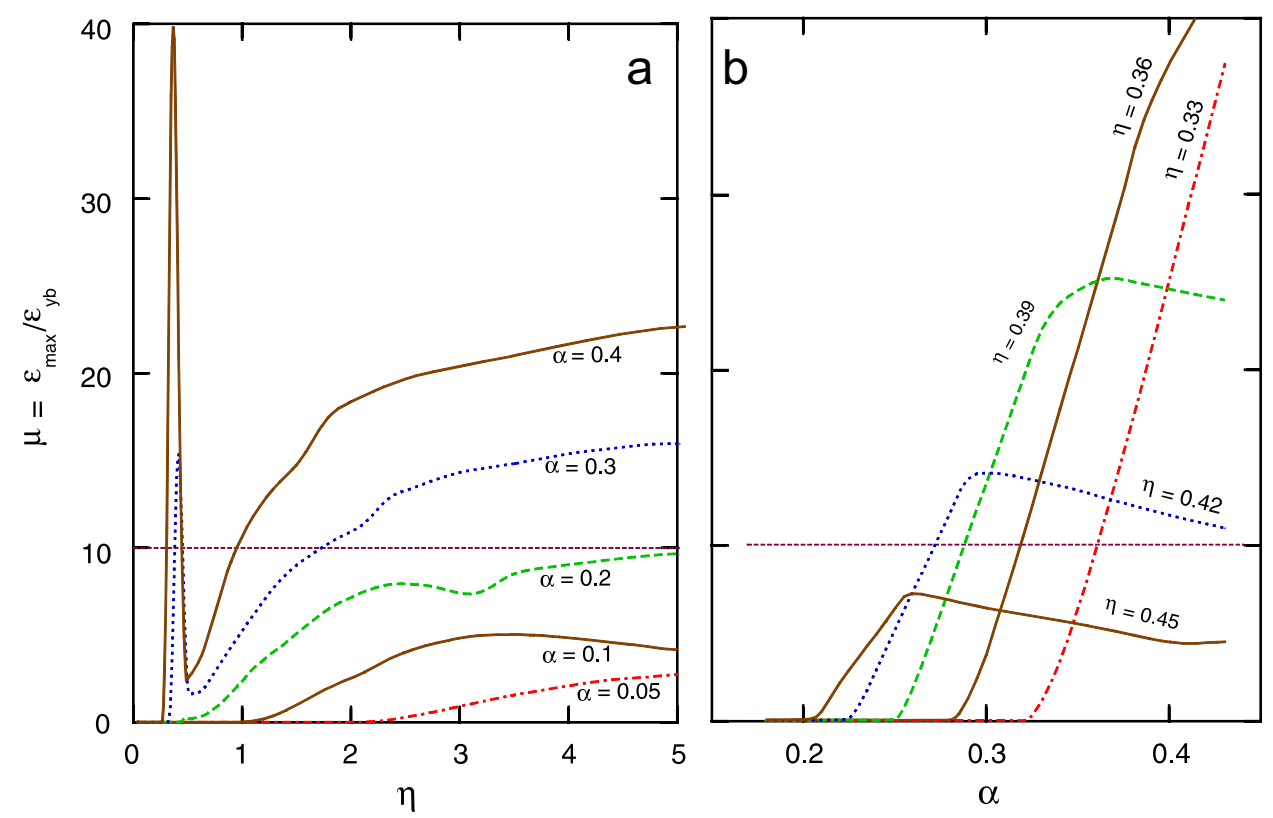

Fig. 10. Ductility versus dimensionless frequency $\eta$ (left) and dimensionless amplitude $\alpha$ (right). 

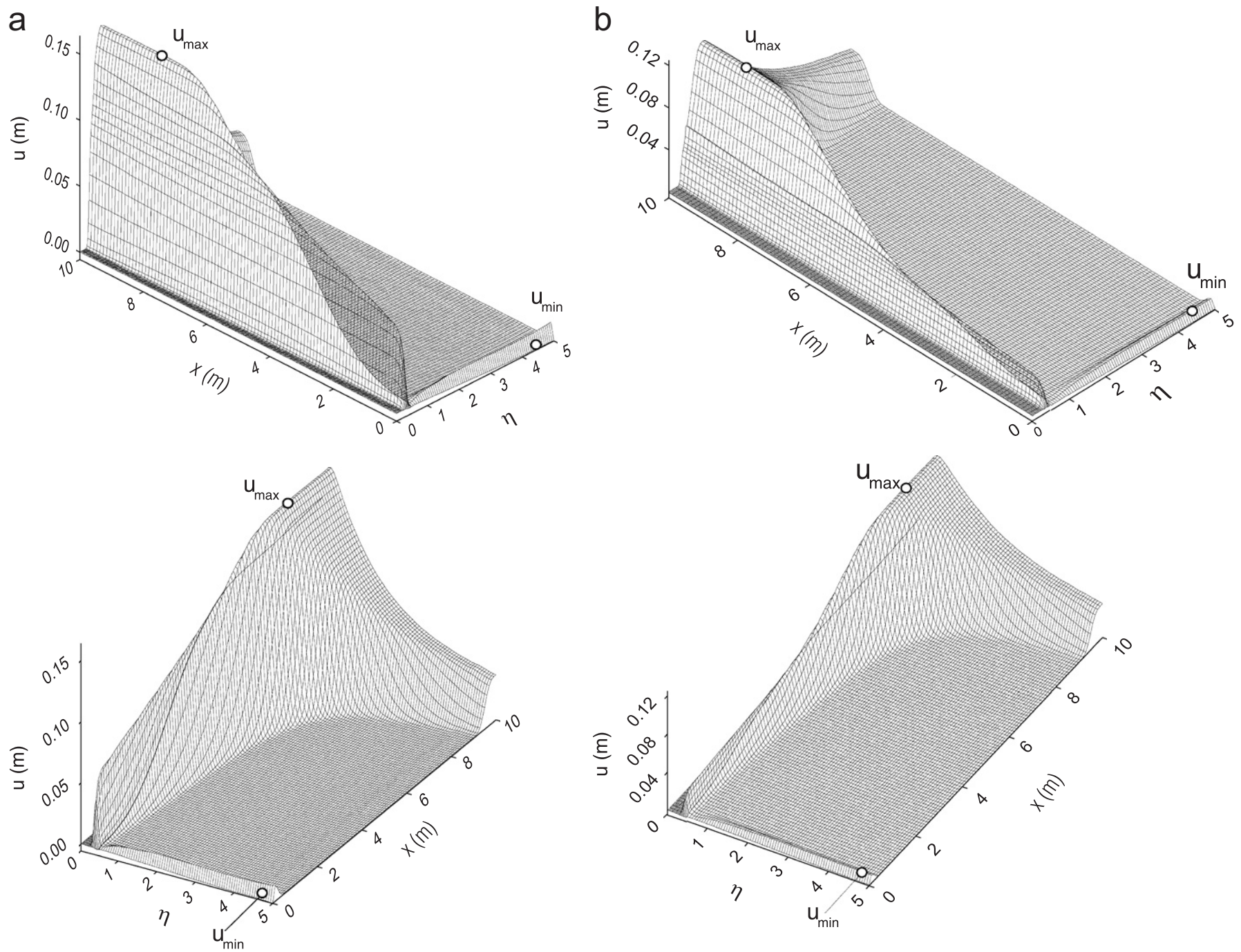

Fig. 11. Permanent displacements along the beam versus dimensionless frequency $\eta$, for dimensionless amplitude (a) $\alpha=0.4$, (b) $\alpha=0.3$, (c) $\alpha=0.2$, (d) $\alpha=0.1$, and (e) $\alpha=0.05$, and for two different view angles.

for higher frequencies and higher strains, because the pulse loses more energy while creating the permanent strains in the first passage. For $\eta=0.45$ and for amplitudes $\alpha>0.41$, because the maximum occurs at some point above B, additional time is needed for the pulse to come to that point.

In Fig. 8 (bottom) the location of the point where the permanent strain is maximum is presented as a function of $\eta$ for $\alpha=0.05,0.1,0.2,0.3$, and 0.4 . It can be seen that the largest strain for $\alpha=0.4,0.3$, and 0.2 first occurs at the bottom, satisfying the condition in Eq. (4.8B2). The condition in Eq. (4.8B2) is for the long pulses $(\eta \leqslant 0.5)$, and so in our examples it cannot be satisfied for $\alpha=0.1$ and 0.05 . For these small amplitudes, the largest strain occurs at point $\mathrm{T}$, which satisfies the condition in Eq. (4.8T).

When the condition in Eq. (4.8T) is satisfied, the largest strain for $\alpha=0.4,0.3$, and 0.2 is at point $\mathrm{T}$. It stays there until it is shifted to point $\mathrm{B}$ for certain higher frequencies, where it stays until $\eta$ increases further. Recall that the condition for the occurrence of permanent strain at the bottom of the beam at the beginning of the first passage, for our examples, is given by $\alpha \eta>C_{\mathrm{B}}=0.2228$ (Eq. (4.8B)). When this condition is satisfied, the entering pulse immediately starts to lose energy, so the effect of the reflection from the top of the beam (Eq. (4.8T)) is diminished. When $\alpha \eta$ is slightly larger than $C_{\mathrm{B}}$, a small permanent strain occurs at the bottom, and the permanent strain at the top is smaller than if there were no permanent strain at the bottom. As the product $\alpha \eta$ increases, the permanent strain at the bottom $\eta$ increases, the larger part of the energy is lost at the bottom, and the attenuation of the permanent strain at the top is more pronounced. Following this trend, for some values of the product $\alpha \eta$ the permanent strain at B becomes larger than the permanent strain at $\mathrm{T}$.

It is interesting to note that the sharp drop from point $\mathrm{T}$ to point $\mathrm{B}$ for $\alpha=0.4,0.3$, and 0.2 occurs for approxi- 

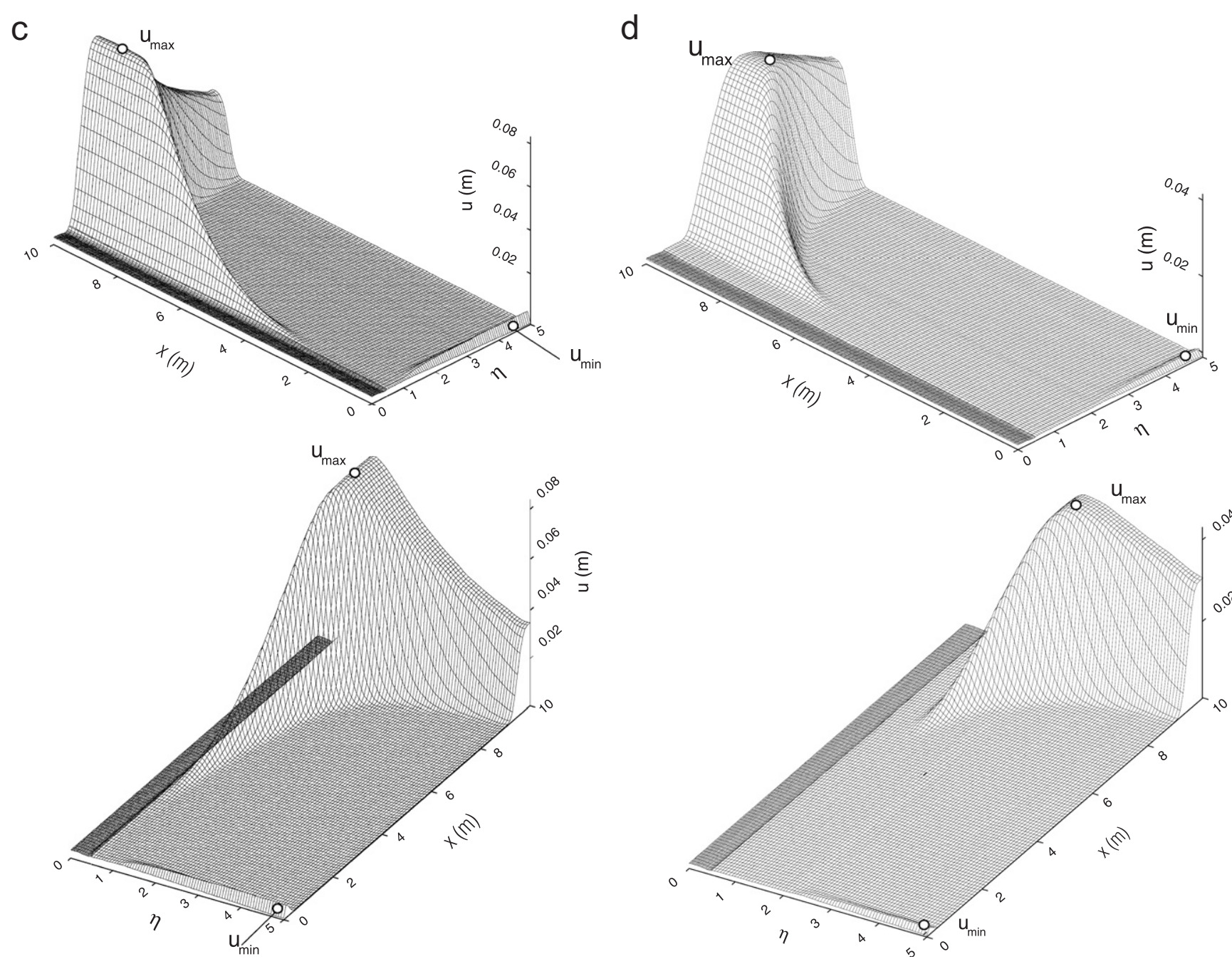

Fig. 11. (Continued)

mately the same values of the product $\alpha \eta$ (see Fig. 8 (bottom)). We have:

- for $\alpha=0.4, \eta_{\mathrm{TB}}=1.91, \alpha \eta_{\mathrm{TB}}=0.764$,

- for $\alpha=0.3, \eta_{\mathrm{TB}}=2.72, \alpha \eta_{\mathrm{TB}}=0.816$,

- for $\alpha=0.2, \eta_{\mathrm{TB}}=4.16, \alpha \eta_{\mathrm{TB}}=0.832$.

This is to be expected, since $\alpha \eta / \pi=\varepsilon_{d_{\max }} / \varepsilon_{\mathrm{yb}}$, where $\varepsilon_{d_{\max }}$ is the peak linear strain associated with the pulse entering the building (see Fig. 1).

In Fig. 5a, the normalized time $\frac{t}{2 H_{\mathrm{b}} / \beta_{\mathrm{b}}}$, when the maximum strain occurs, is shown versus $\eta$. It should be emphasized that there is no connection of this plot with the plot in Fig. 8, which describes the point with maximum strain at the end of the analysis. Fig. 5a shows the time at which the maximum strain occurs, not the strain at the end of the analysis. This difference is illustrated in Fig. 9, where the strain at the bottom point $4(x=\Delta x)$ for $\alpha=0.4$ and $\eta=1$ is shown versus time. It can be seen that in the beginning this point experiences large strain $(\varepsilon=-0.41)$, while at the end of the analysis the strain is small $(\varepsilon=0.05)$.
A sudden drop in the curves can be noticed also in Fig. 5a, analogous to those in Fig. 8 (bottom) as follows:

- for $\alpha=0.4, \eta_{\mathrm{TB}}=0.89, \alpha \eta_{\mathrm{TB}}=0.356$,

- for $\alpha=0.3, \eta_{\mathrm{TB}}=1.19, \alpha \eta_{\mathrm{TB}}=0.357$,

- for $\alpha=0.2, \eta_{\mathrm{TB}}=1.88, \alpha \eta_{\mathrm{TB}}=0.376$.

Similar conclusions can be drawn here as for the example in the previous paragraph.

Fig. 10a and $\mathrm{b}$ present the ductility ratio (maximum permanent strain normalized by the yielding strain) in the building. Fig. 10a shows ductility versus $\eta(0.03 \leqslant \eta \leqslant 5)$ for five dimensionless excitation amplitudes $\alpha=0.05 ; 0.1 ; 0.2$; $0.3 ; 0.4$. Fig. $10 \mathrm{~b}$ shows the ductility versus $\alpha$ in the vicinity of the largest strain (see Fig. 6a).

If our hypothetical beam is made of a material that can experience maximum ductility $\mu_{0}=10$, then Fig. 10a implies failure for the following values of $\alpha$ and $\eta$ in the range of the parameters we consider: for $\alpha=0.4(0.3 \leqslant \eta \leqslant$ $0.43) \cup \eta \geqslant 0.95$, and for $\alpha=0.3(0.38 \leqslant \eta \leqslant 0.44) \cup \eta \geqslant 1.73$. The beam will not fail for dimensionless frequencies 


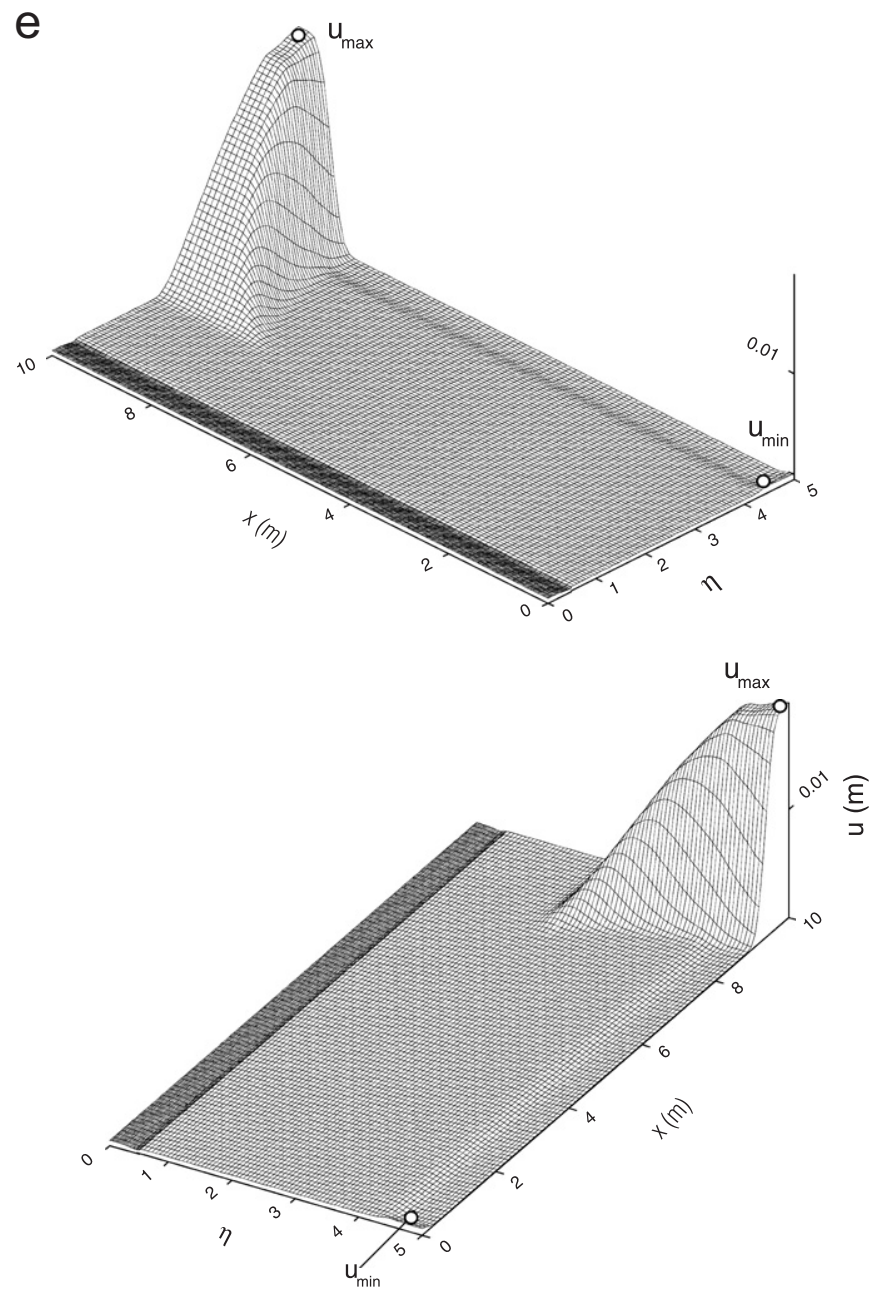

Fig. 11. (Continued)

outside the above intervals, and also for all values of $\alpha \leqslant 0.2$. From Fig. 10b, it can be seen that the same beam will fail for the following values of $\alpha$ and $\eta: \eta=0.33$, $\alpha \geqslant 0.36 ; \eta=0.36, \alpha \geqslant 0.32 ; \eta=0.39, \alpha \geqslant 0.29$; and $\eta=0.42$, $\alpha \geqslant 0.27$. This beam will not fail for smaller amplitudes than the ones given above, and for $\eta=0.45$, for all $\alpha$ in the range we considered here $(0.18 \leqslant \alpha \leqslant 0.43)$.

All of the above discussed trends can be summarized by three-dimensional plots of permanent displacements $u(x, \eta)$ and permanent strains $\varepsilon(x, \eta)$, for $\alpha=0.4,0.3,0.2,0.1$, and 0.05 . To show the peaks occurring for $\eta<0.5$ (see Fig. 6) with better detail, the interval $0.03 \leqslant \eta \leqslant 0.5$ is discretized with a three times denser grid in the $\eta$ direction than the interval $0.5 \leqslant \eta \leqslant 5$. Fig. 11 shows the distribution of permanent displacements, while Fig. 12 shows the distribution of permanent strains. Tables 1 and 2 summarize the position and the extreme amplitudes shown in Figs. 11 and 12.

In Figs. 13 and 14, the displacements along the normalized height of beam $\chi=\frac{x}{H_{\mathrm{b}}}$ versus the dimensionless time $\tau=\beta_{\mathrm{b}} t /\left(2 H_{\mathrm{b}}\right)$ are shown. In this way, $\tau$ becomes an integer when the pulse hits the building-soil interface. In Fig. 13 the dimensionless frequency is $\eta=3$ (short pulse), and in Fig. 14 it is $\eta=0.41$ (long pulse). The yielding strain of the beam for the examples shown in these figures is $\varepsilon_{\mathrm{yb}}=0.02$.

In Fig. 13a, the propagation of a half-sine pulse with dimensionless amplitude $\alpha=0.03$ and dimensionless frequency $\eta=3$ is shown. For this small $\alpha$, the response of the beam remains linear for all time. In Fig. 13b, the propagation of the pulse with $\alpha=0.1$ is illustrated, and in Fig. 13c the propagation of a pulse with the amplitude $\alpha=0.3$ is shown. As can be seen in Fig. 13a, the linear pulse after reflection from the building-soil interface loses some energy due to radiation and changes its sign. After the second reflection from this interface $(\tau=2)$, a substantial part of the pulse is still in the building. This ceases to be so in Fig. 13b. Immediately after the creation of the non-linear zone at the top, a part of the energy in the pulse is "absorbed" to form large permanent strains at the top, and only a small part of the pulse energy propagates downward. After reaching the building-soil interface, this remaining pulse loses additional energy to radiation and to the need to overcome the permanent strain at the bottom, so that after the first reflection from the building-soil interface the pulse almost vanishes. In Fig. 13c, the permanent strain at the bottom occurs in the very beginning, so that the part of the pulse propagating up is weaker. After reflecting from the top, a large permanent strain is formed, and almost all of the energy of the pulse is lost.

In Fig. 14a for $\alpha=0.2$ the response of the beam is linear for all time. For $\alpha=0.25$ (Fig. 14b) the first permanent strain appears after the first reflection from the buildingsoil interface $(\tau=1)$. With increasing amplitude $\alpha$, this permanent strain increases, but for this range of dimensionless frequencies this occurs at time $\tau=1$. In Fig. 14c, the response is illustrated for $\alpha=0.3$.

To further assist in interpretation of the results presented in Figs. 13 and 14, Table 3 summarizes the maximum and minimum amplitudes of displacements, at the building-soil interface, $u_{0}$, and in the building, $u$ (Table 4).

\section{Conclusions}

In this paper, the occurrence, development, and amplitudes of permanent strains and displacements have been studied for a one-dimensional soil-structure interaction model consisting of an elasto-plastic shear beam on an elastic foundation. Excitation for five dimensionless amplitudes of a half-sinusoidal displacement pulse with strong ground motion $(\alpha=0.05 ; 0.1 ; 0.2 ; 0.3$; and 0.4$)$ in a wide range of dimensionless frequencies $(0.03 \leqslant \eta \leqslant 5)$ were analyzed.

It has been found that for large ground displacement pulses (large $\alpha$ ) the maximum permanent strains occur mainly at the interface of the building with the soil, while for smaller amplitudes of pulses permanent strains occur closer to the top of the building. Three zones of the permanently deformed beam can be distinguished: (1) A 

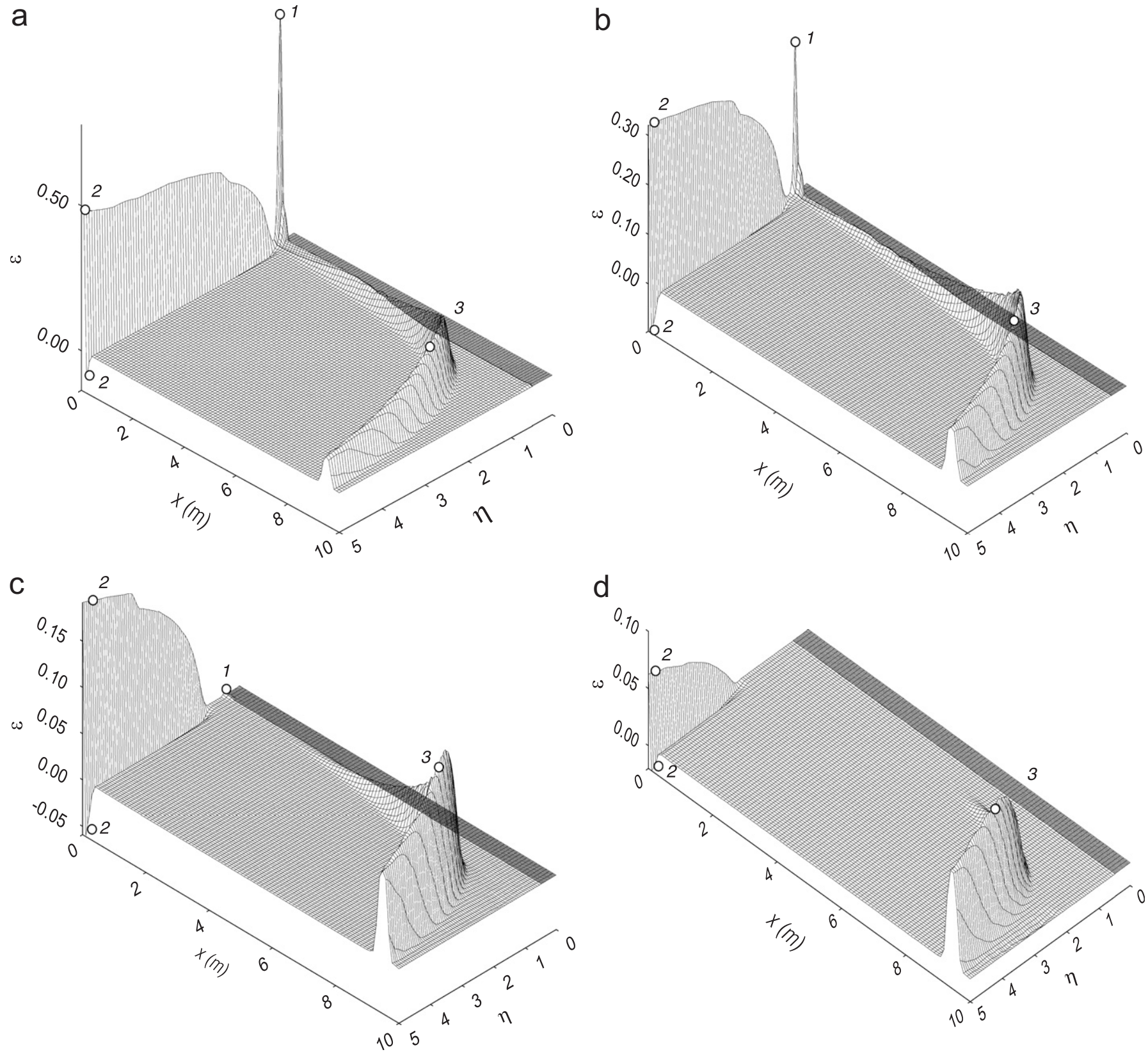

Fig. 12. Permanent strain along the beam versus dimensionless frequency $\eta$, for dimensionless pulse amplitude (a) $\alpha=0.4$, (b) $\alpha=0.3$, (c) $\alpha=0.2$, (d) $\alpha=0.1$, and (e) $\alpha=0.05$.

permanently deformed zone at the bottom; (2) An intermediate zone, which is not deformed at its bottom part and is deformed in the top part; and (3) A nondeformed zone at the top of the beam. The occurrence and the development of these zones depend upon the dimensionless excitation amplitudes and the dimensionless frequency, and in particular on the conditions that lead to the occurrence of the first permanent strain (see Eqs. (4.8B), (4.8T), and (4.8B2) in the text). For large and long strong motion pulses $(\eta \leqslant 0.5$; first the condition in Eq. (4.8B2) is relevant), only zones 1 and 3 are present in the beam. For large amplitudes and short strong-motion pulses, all three zones develop and are present. For smaller excitation amplitudes (when the condition in Eq. (4.8B2) cannot be satisfied for long pulses, and when the condition in Eq. (4.8T) is satisfied) only zones 2 and 3 exist in the beam. For larger values of $\eta$ (when the condition in Eq. $(4.8 \mathrm{~B})$ is satisfied) all three zones exist.

The situation is similar for the occurrence of the maximum strains. For large and long pulses, maximum strain is located at the bottom of the building, and as the pulses become shorter peak strains occur at higher positions in the building. For some high frequencies of excitation, the maximum strain again appears at the bottom of the building because the loss of energy due to the development of the permanent strain at the bottom 
overcomes the effects of the wave reflections from the top of the building.

Creation of large permanent deformation zones in the building by the incident waves absorbs some or most of the incident wave energy and can reduce or eliminate further wave propagation and the associated

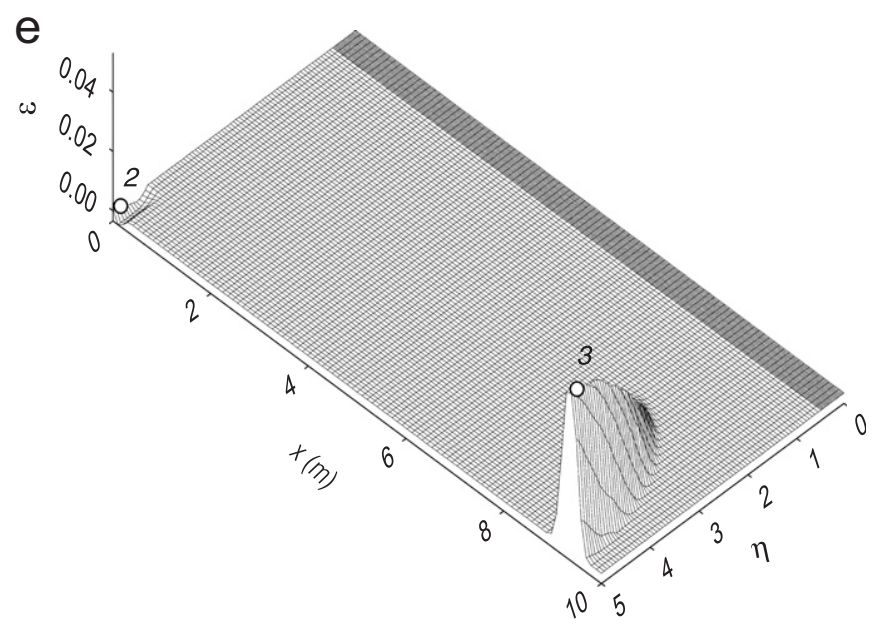

Fig. 12. (Continued) energy transport. To the extent that the locations of the plastic deformation zones can be controlled by the design process, absorption of the incident wave energy by structural members may become a new and powerful tool for the performance-based design. To take advantage of such possibilities, the governing differential equations must be solved by the wave propagation method.

Examples studied in this paper show that for excitation of structures by large, near-field displacement pulses, failure can occur anywhere in the building before the incident wave has completed its first travel from the foundation to the top of the building and back to the foundation $\left(2 H_{\mathrm{b}} / \beta_{\mathrm{b}}\right)$. Because this travel time is shorter (by $\frac{1}{2}$ ) than the natural period of the structure on the fixed base, it is seen that the common response spectrum method of analysis (based on the vibrational formulation of the solution) cannot provide the required details for the design of structures for such excitation.

\section{Appendix A. Equivalent shear modulus at interface point 3}

To derive the equivalent shear modulus at the interface, which can be used in finite difference computation,

Table 1

Maximum and minimum displacements, $u_{\max }$ and $u_{\min }$, and their locations, $\left(\eta_{\max }, x_{\max }\right)$ and $\left(\eta_{\min }, x_{\min }\right)$, in Fig. 11a-e

\begin{tabular}{llllllll}
\hline$\alpha$ & Fig. no. & $\eta_{\max }$ & $x_{\max }(\mathrm{m})$ & $u_{\max }(\mathrm{cm})$ & $\eta_{\min }$ & $x_{\min }(\mathrm{m})$ & $u_{\min }(\mathrm{cm})$ \\
\hline 0.40 & $11 \mathrm{a}$ & 0.68 & 7.56 & 8.48 & 16.704 & 4.40 & 0.36 \\
0.30 & $11 \mathrm{~b}$ & 0.89 & 8.88 & 12.606 & 4.82 & 0.36 \\
0.20 & $11 \mathrm{c}$ & 1.31 & 9.34 & 4.371 & 4.82 & 0.36 & -0.503 \\
0.10 & $11 \mathrm{~d}$ & 2.63 & 9.80 & 1.982 & 4.97 & 0.30 & -0.338 \\
0.05 & $11 \mathrm{e}$ & 4.97 & & & 4.58 & 0.30 & -0.128 \\
\hline
\end{tabular}

Table 2

Maximum and minimum strains, $\varepsilon_{\max }$ and $\varepsilon_{\min }$, and their locations, $\left(\eta_{\max }, x_{\max }\right)$ and $\left(\eta_{\min }, x_{\min }\right)$, in the three zones in Fig. 12a, b, c

\begin{tabular}{|c|c|c|c|c|c|c|c|c|}
\hline$\alpha$ & Fig. no. & Zone \# & $\eta_{\max }$ & $x_{\max }(\mathrm{m})$ & $\varepsilon_{\max }$ & $\eta_{\min }$ & $x_{\min }(\mathrm{m})$ & $\varepsilon_{\min }$ \\
\hline 0.4 & $12 \mathrm{a}$ & $\begin{array}{l}1 \\
2 \\
3\end{array}$ & $\begin{array}{l}0.36 \\
4.97 \\
1.76\end{array}$ & $\begin{array}{l}0.0 \\
0.0 \\
8.48\end{array}$ & $\begin{array}{l}0.79584 \\
0.50137 \\
0.23798\end{array}$ & 4.97 & 0.10 & -0.1364 \\
\hline 0.3 & $12 b$ & $\begin{array}{l}1 \\
2 \\
3\end{array}$ & $\begin{array}{l}0.41 \\
4.91 \\
2.00\end{array}$ & $\begin{array}{l}0.0 \\
0.0 \\
8.63\end{array}$ & $\begin{array}{l}0.30714 \\
0.32172 \\
0.20205\end{array}$ & 4.91 & 0.10 & -0.0951 \\
\hline 0.2 & $12 \mathrm{c}$ & $\begin{array}{l}1 \\
2 \\
3\end{array}$ & $\begin{array}{l}0.46 \\
4.79 \\
2.42\end{array}$ & $\begin{array}{l}0.0 \\
0.0 \\
8.83\end{array}$ & $\begin{array}{l}0.00495 \\
0.19734 \\
0.15989\end{array}$ & 4.79 & 0.10 & -0.0592 \\
\hline 0.1 & $12 \mathrm{~d}$ & $\begin{array}{l}1 \\
2 \\
3\end{array}$ & $\begin{array}{l}- \\
4.97 \\
3.44\end{array}$ & $\begin{array}{l}- \\
0.0 \\
9.14\end{array}$ & $\begin{array}{l}- \\
0.06243 \\
0.10097\end{array}$ & 4.97 & 0.10 & -0.0199 \\
\hline 0.05 & $12 \mathrm{e}$ & $\begin{array}{l}1 \\
2 \\
3\end{array}$ & $\begin{array}{l}- \\
4.97 \\
4.97\end{array}$ & $\begin{array}{l}- \\
0 . \\
9.34\end{array}$ & $\begin{array}{l}- \\
0.00079 \\
0.05447\end{array}$ & 4.49 & 0.05 & -0.0039 \\
\hline
\end{tabular}

Zone $1: \eta<0.5 \cup x \leqslant 10 \mathrm{~m}$, zone $2: 0.5 \leqslant \eta \leqslant 5 \cup x \leqslant 1 \mathrm{~m}$, and zone $3: 0.5 \leqslant \eta \leqslant 5 \cup x>1 \mathrm{~m}$. 
a
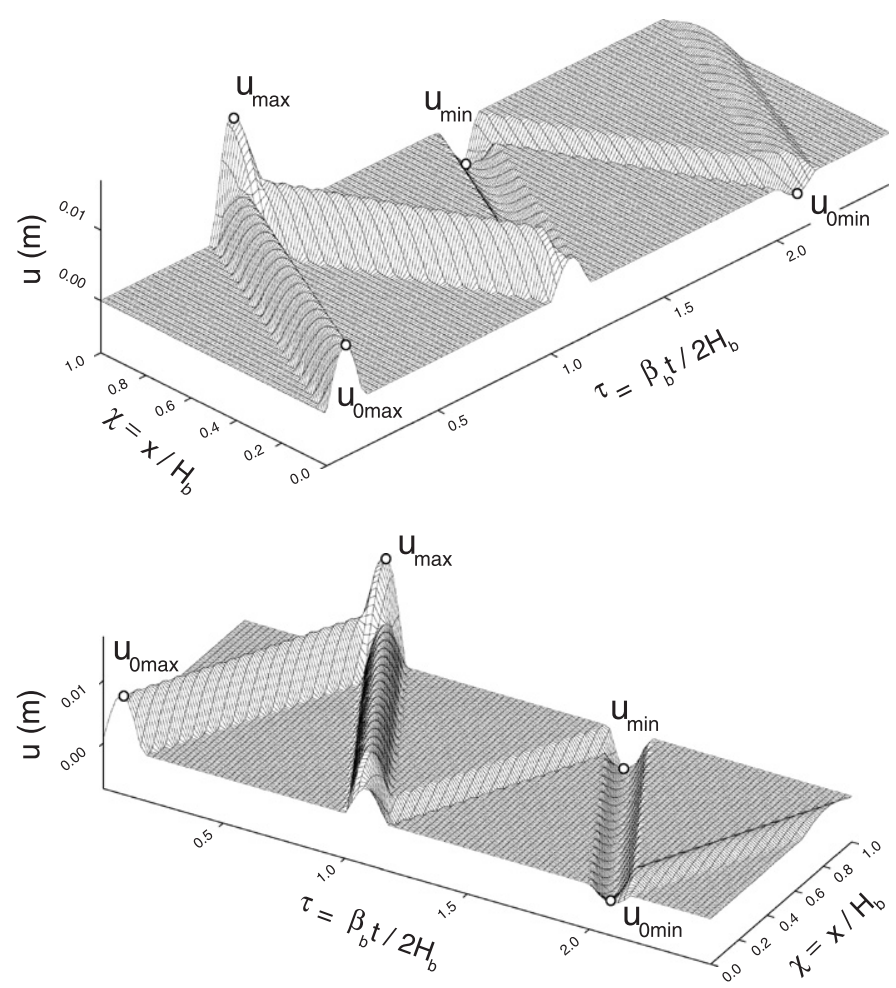

C b
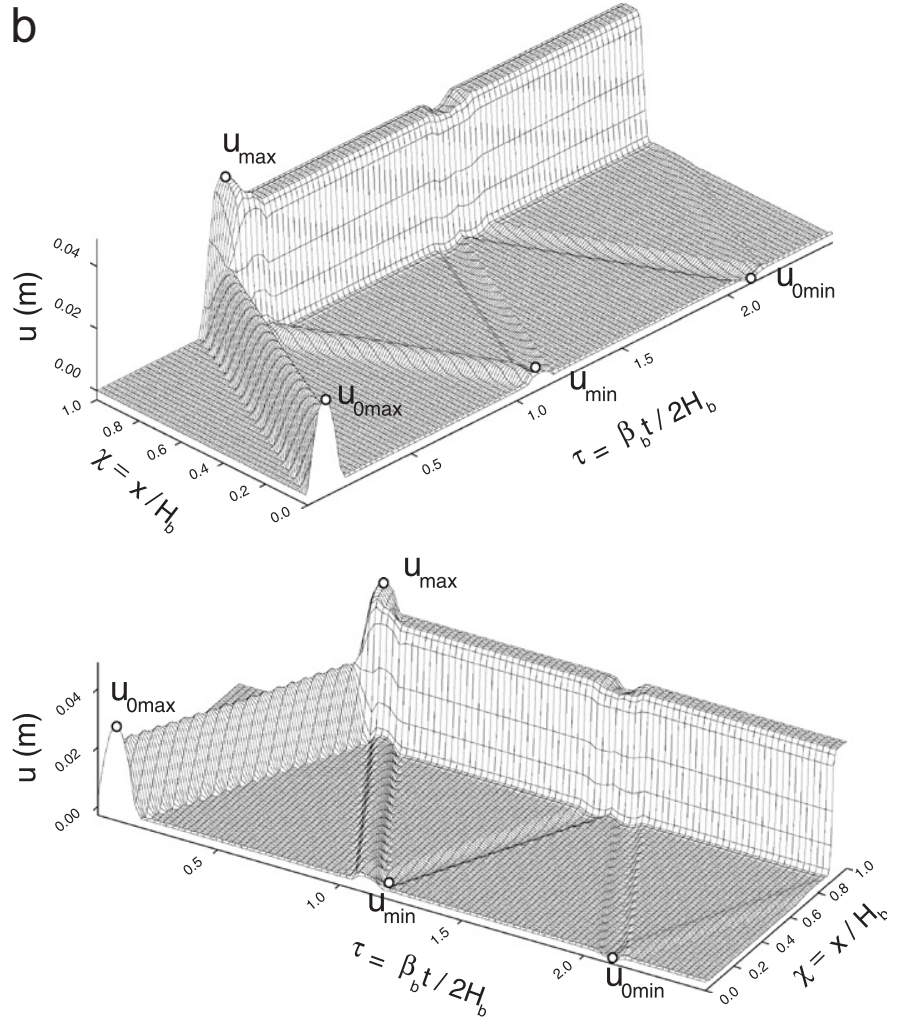
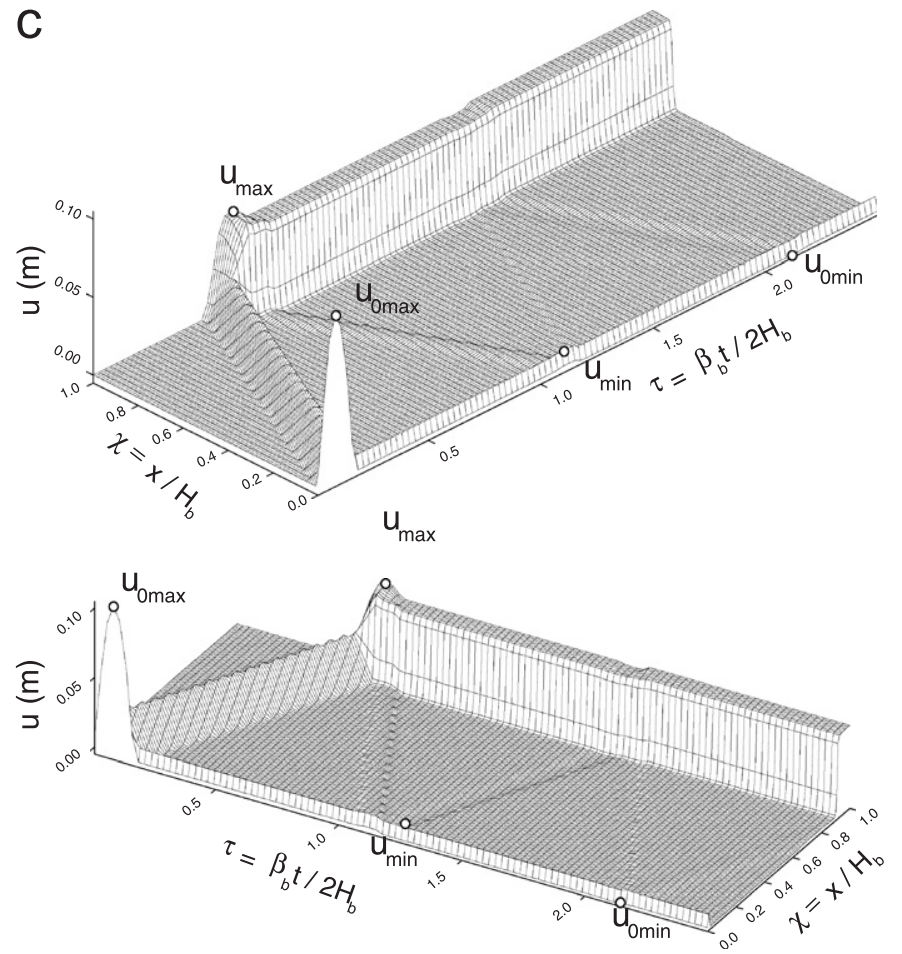

Fig. 13. Displacements along the normalized length of the beam, $\chi=x / H_{\mathrm{b}}$, versus normalized time $\tau=\beta_{\mathrm{b}} t / 2 H_{\mathrm{b}}$, for dimensionless pulse amplitude (a) $\alpha=0.03$, (b) $\alpha=0.1$, (c) $\alpha=0.3$ and dimensionless frequency $\eta=3$ (shown for two different view angles).

we introduce two dummy points: (1) point $\overline{2}$, above point 3 , at distance $\Delta x_{\mathrm{s}}$, associated with the material properties of the soil; and (2) point $\overline{4}$, below point 3 , at distance $\Delta x_{\mathrm{b}}$, having the material properties of the building.
The numerical representation of continuity of the stresses and the displacements at point 3 is

$\mu_{\mathrm{b}} \frac{u_{4}-u_{\overline{4}}}{2 \Delta x_{\mathrm{b}}}=\mu_{\mathrm{s}} \frac{u_{\overline{2}}-u_{2}}{2 \Delta x_{\mathrm{s}}}$, 

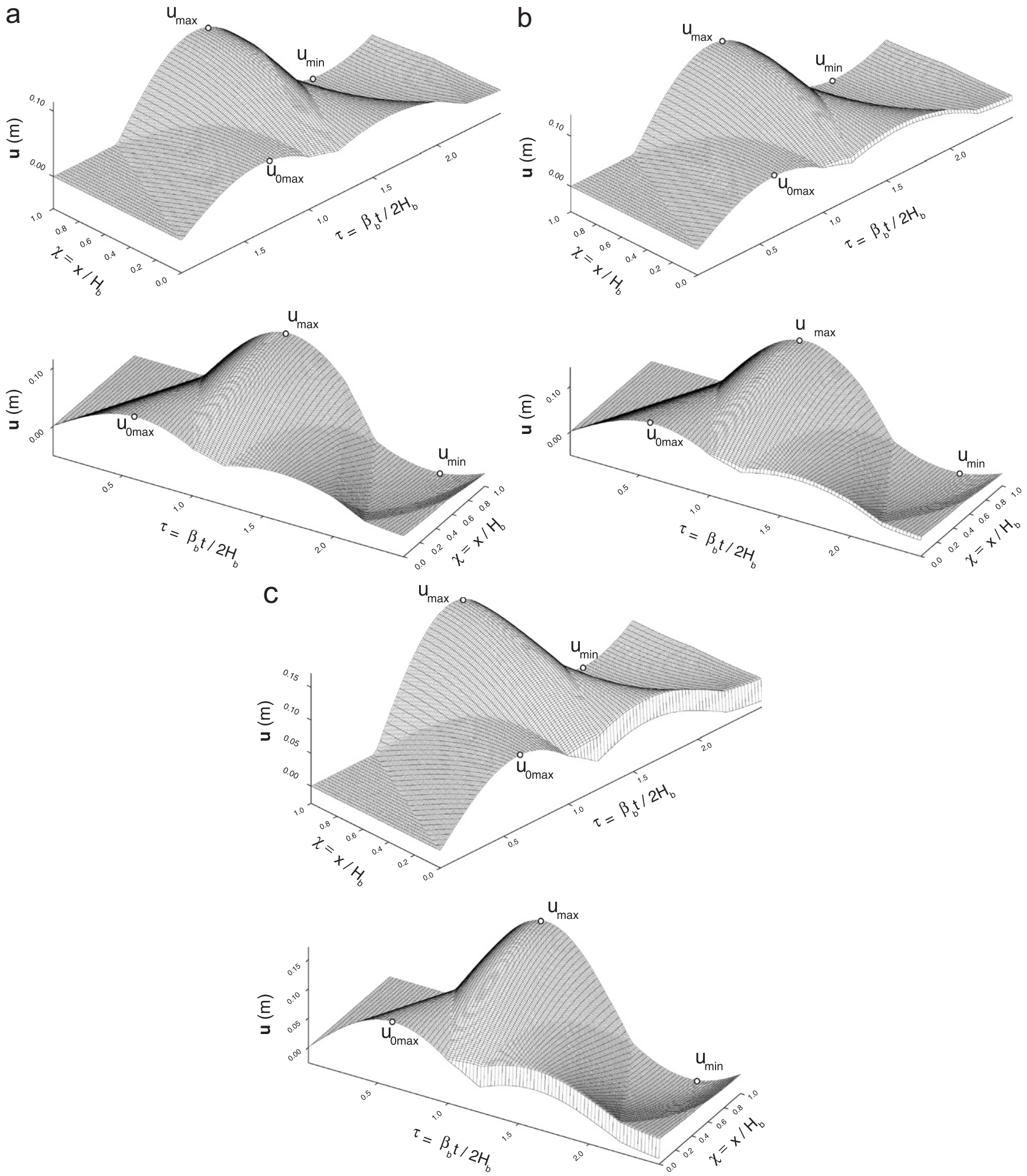

Fig. 14. Displacements along the normalized length of the beam, $\chi=x / H_{\mathrm{b}}$, versus normalized time $\tau=\beta_{\mathrm{b}} t / 2 H_{\mathrm{b}}$, for dimensionless pulse amplitude (a) $\alpha=0.2$, (b) $\alpha=0.25$, (c) $\alpha=0.3$ and dimensionless frequency $\eta=0.41$ (shown for two different view angles).

$\frac{u_{4}+u_{\overline{4}}}{2}=\frac{u_{2}+u_{\overline{2}}}{2}$

Solving for $u_{\overline{4}}$ (or $u_{\overline{2}}$ ) and substituting the solution into the left-hand side (or right-hand side) of (A.1), the stress at the 
Table 3

$u_{\text {soil }}$-amplitude of the incoming wave in the soil; $u_{0_{\max }}$ and $u_{0_{\min }}$-maximum and minimum displacements of the building-soil interface and the corresponding $\tau_{0_{\max }}$ and $\tau_{0_{\min }}$, where these extrema occur; and $u_{\max }$ and $u_{\min }-$ maximum and minimum displacements and their locations at ( $\left.\tau_{\max }, \chi_{\max }\right)$ and $\left(\tau_{\min }, \chi_{\min }\right)$, respectively (see Figs. 13 and 14$)$

\begin{tabular}{|c|c|c|c|c|c|c|}
\hline$\alpha$ & Fig. no. & $u_{\text {soil }}(\mathrm{m})$ & $\begin{array}{l}u_{0 \max }(\mathrm{m}) \\
\tau_{0 \max }\end{array}$ & $\begin{array}{l}u_{0 \min }(\mathrm{m}) \\
\tau_{0 \min }\end{array}$ & $\begin{array}{l}u_{\max }(\mathrm{m}) \\
\left(\tau_{\max }, \chi_{\max }\right)\end{array}$ & $\begin{array}{l}u_{\min }(\mathrm{m}) \\
\left(\tau_{\min }, \chi_{\min }\right)\end{array}$ \\
\hline 0.03 & $13 \mathrm{a}$ & 0.006 & $\begin{array}{l}0.00857 \\
0.086\end{array}$ & $\begin{array}{c}-0.00209 \\
2.091\end{array}$ & $\begin{array}{l}0.017 \\
(0.581,0.995)\end{array}$ & $\begin{array}{l}-0.00732 \\
(1.584,0.995)\end{array}$ \\
\hline 0.10 & $13 b$ & 0.02 & $\begin{array}{l}0.02959 \\
0.086\end{array}$ & $\begin{array}{c}-0.00116 \\
2.091\end{array}$ & $\begin{array}{l}0.04890 \\
(0.594,0.995)\end{array}$ & $\begin{array}{l}-0.00341 \\
(1.14,0.071)\end{array}$ \\
\hline 0.30 & $13 \mathrm{c}$ & 0.06 & $\begin{array}{l}0.10497 \\
0.086\end{array}$ & $\begin{array}{c}-0.00332 \\
2.117\end{array}$ & $\begin{array}{c}0.10497 \\
(0.086,0.0)\end{array}$ & $\begin{array}{l}-0.00516 \\
(1.165,0.091)\end{array}$ \\
\hline 0.20 & $14 \mathrm{a}$ & 0.04 & $\begin{array}{l}0.05714 \\
0.619\end{array}$ & - & $\begin{array}{l}0.11428 \\
(1.114,0.995)\end{array}$ & $\begin{array}{l}-0.04955 \\
(2.117,0.995)\end{array}$ \\
\hline 0.25 & $14 \mathrm{~b}$ & 0.05 & $\begin{array}{l}0.07142 \\
0.619\end{array}$ & - & $\begin{array}{l}0.14285 \\
(1.114,0.995)\end{array}$ & $\begin{array}{l}-0.05075 \\
(2.117,0.995)\end{array}$ \\
\hline 0.30 & $14 \mathrm{c}$ & 0.06 & $\begin{array}{l}0.08571 \\
0.619\end{array}$ & - & $\begin{array}{l}0.17142 \\
(1.114,0.995)\end{array}$ & $\begin{array}{l}-0.02619 \\
(2.117,0.995)\end{array}$ \\
\hline
\end{tabular}

Table 4

To interpret the loss of energy, as the pulse creates and then propagates through the zone of permanent strain, and because the pulse for $\eta=3$ is shorter than $H_{\mathrm{b}}$, the amplitudes of the response at $\chi=0.5$ for five consecutive passes are reported in this table. The amplitude of the permanent displacement at the end is shown in the last column (see Fig. 13b, c)

\begin{tabular}{|c|c|c|c|c|c|c|c|}
\hline$\alpha$ & Fig. no. & $\begin{array}{l}\tau_{1} \\
A_{1}(\mathrm{~cm})\end{array}$ & $\begin{array}{l}\tau_{2} \\
A_{2}(\mathrm{~cm})\end{array}$ & $\begin{array}{l}\tau_{3} \\
A_{3}(\mathrm{~cm})\end{array}$ & $\begin{array}{l}\tau_{4} \\
A_{4}(\mathrm{~cm})\end{array}$ & $\begin{array}{l}\tau_{5} \\
A_{5}(\mathrm{~cm})\end{array}$ & $\begin{array}{l}\tau_{\text {end }} \\
A_{\text {end }}(\mathrm{cm})\end{array}$ \\
\hline 0.1 & $13 b$ & $\begin{array}{l}0.3299 \\
2.44517\end{array}$ & $\begin{array}{l}0.8426 \\
0.38029\end{array}$ & $\begin{array}{c}1.3299 \\
-0.24846\end{array}$ & $\begin{array}{c}1.8477 \\
-0.23245\end{array}$ & $\begin{array}{l}2.3350 \\
0.03569\end{array}$ & $\begin{array}{r}2.5000 \\
-0.0468\end{array}$ \\
\hline 0.3 & $13 \mathrm{c}$ & $\begin{array}{l}0.3274 \\
2.95771\end{array}$ & $\begin{array}{c}0.8680 \\
-0.05625\end{array}$ & $\begin{array}{c}1.3553 \\
-0.51400\end{array}$ & $\begin{array}{c}1.8680 \\
-0.42024\end{array}$ & $\begin{array}{c}2.3553 \\
-0.22527\end{array}$ & $\begin{array}{c}2.5000 \\
-0.29591\end{array}$ \\
\hline
\end{tabular}

interface is obtained as

$\sigma_{3}=\frac{\mu_{\mathrm{b}} \mu_{\mathrm{s}}}{\mu_{\mathrm{b}} \Delta x_{\mathrm{s}}+\mu_{\mathrm{s}} \Delta x_{\mathrm{b}}}\left(u_{4}-u_{2}\right)$.

For linear analysis, one can use the stress (A.3) in the computations, but for non-linear analysis, when the material at point 4 yields, Eq. (A.3) is not convenient because the shear modulus $\mu_{\mathrm{b}}$ varies in time, and if at some instant it becomes zero Eq. (A.3) gives zero stress at the interface.

\section{Appendix B. The largest permanent strain in the beam}

For the range of the parameters considered in this paper, the largest permanent strain occurs for $\alpha=0.4$ and $\eta=0.36$, at point $4(x=\Delta x)$. The duration of the pulse in this case is $t_{\mathrm{d}}=0.27778 \mathrm{~s}$. The strain at point 4 is computed by iteration from

$\varepsilon_{4}^{k+1}=\varepsilon_{4}^{k}+\frac{\Delta t}{2 \Delta x}\left(v_{5}^{k}-v_{3}^{k}\right)+\frac{\Delta t^{2}}{2 \rho \Delta x^{2}}\left(\sigma_{5}^{k}-2 \cdot \sigma_{4}^{k}+\sigma_{3}^{k}\right)$.
Because of the assumption that the yielding strain at the interface point is $\varepsilon_{\mathrm{y} 3}=\frac{\mu_{\mathrm{b}}}{\mu_{3}} \cdot \varepsilon_{\mathrm{yb}}$, the stresses $\sigma_{5}, \sigma_{4}$, and $\sigma_{3}$ are essentially equal during all time in this analysis, and so the third term in the right-hand side of Eq. (B.1) can be omitted. The permanent strain obtained in this way is 0.75 , while including the last term in the RHS of Eq. (B.1) would give 0.796 .

The bottom of the beam starts to yield at time $t=0.2 \mathrm{~s}$, when the reflected wave from the top reaches the bottom again. In the beginning, there is constructive interference from two positive strains:

- the first positive strain, with maximum amplitude $A_{\varepsilon} \cdot k_{t}=\frac{A \pi}{\beta_{\mathrm{b}} t_{\mathrm{d}}} \cdot k_{t}$, coming from above (from the reflected pulse), and

- the second positive strain, arriving from the linear halfspace below, with amplitude $-A_{\varepsilon} \cdot k_{t} \cos \pi \frac{t}{t_{\mathrm{d}}}=$ $A_{\varepsilon} \cdot k_{t} \sin \pi \frac{t_{\mathrm{d}}-t}{t_{\mathrm{d}}}$.

After reflecting from the bottom of the building, the reflected strain is still positive, but it has a reduced 
amplitude $A_{\varepsilon}^{\prime}=-k_{r}^{b-b} \cdot k_{t} \cdot A_{\varepsilon}$, where $k_{r}^{b-b}=\left(1-\beta_{\mathrm{s}} / \beta_{\mathrm{b}}\right) /$ $\left(1+\beta_{\mathrm{s}} / \beta_{\mathrm{b}}\right)$.

The velocity at point 3 (see Fig. 1) continues to follow the linear motion in the half-space. At the same time, the velocity at point 5 experiences a jump and then remains constant as long as the neighboring points 4 and 6 are in the plastic state. When point 6 starts to unload, the velocity at point 5 decreases and follows the input velocity from the ground. At the time $t=t_{\mathrm{d}}+2 \frac{\Delta x_{\mathrm{s}}}{\beta_{\mathrm{s}}}+2 \frac{\Delta x_{\mathrm{b}}}{\beta_{\mathrm{b}}} \approx t_{\mathrm{d}}$, the whole pulse, arriving from the ground, has passed points 5 and 3 , and the velocities at these two points remain equal until the end of the analysis.

\section{References}

[1] Trifunac MD, Todorovska MI. Recording and interpreting earthquake response of full-scale structures. In: Erdik $M$ et al., editor. Proceedings of NATO workshop on strong motion instrumentation for civil engineering structures, June 2-5, 1999, Instanbul, Turkey. Dordrecht: Kluwer Academic Publishers; 2001. p. 131-55.

[2] Biot MA. Vibrations of building during earthquake. In: Transient oscillations in elastic systems. PhD thesis No. 259, Aeronautics Department, California Institute of Technology, Pasadena, CA; 1932 [chapter 2].

[3] Biot MA. Theory of elastic systems vibrating under transient impulse with an application to earthquake-proof building. Proc Nat Acad Sci 1933;19(2):262-8.

[4] Biot MA. Theory of vibration of buildings during earthquake. Z Angew Mat Mech 1934;14(4):213-23.

[5] Trifunac MD. 70-th Anniversary of Biot spectrum, 23rd annual ISET lecture. Indian Society of Earthquake Technology, vol. 1(40), No. 1; 2003. p. $19-50$.

[6] Trifunac MD. Power design method. In: Proceedings of earthquake engineering in the 21 st century to mark 40th anniversary of IZIISSkopje, August 28-September 1, 2005, Skopje and Ohrid, Macedonia; 2005.

[7] Kanai K. Some problems of seismic vibration of structures. In: Proceedings of the third world conference on earthquake engineering, New Zealand, vol. II; 1965. p. 260-75.

[8] Ivanović SS, Trifunac MD, Novikava EI, Gladkov AA, Todorovska MI. Instrumented 7-story reinforced concrete building in Van Nuys, California: ambient vibration surveys following the damage from the 1994 Northridge earthquake. Report No. CE 99-03, Department of Civil Engineering, University of Southern California, Los Angeles, California; 1999 (can be downloaded from: $\langle$ http://www.usc.edu/ dept/civil_eng/Earthquake_eng/ $>$ ).

[9] Ivanović SS, Trifunac MD, Novikava EI, Gladkov AA, Todorovska MI. Ambient vibration tests of a seven-story reinforced concrete building in Van Nuys, California, damaged by the 1994 Northridge earthquake. Soil Dyn Earthquake Eng 2000;19(6):391-411.

[10] Todorovska MI, Ivanovic SS, Trifunac MD. Wave propagation in a seven-story reinforced concrete building, Part I: theoretical models. Soil Dyn Earthquake Eng 2001;21(3):211-23.

[11] Todorovska MI, Ivanovic SS, Trifunac MD. Wave propagation in a seven-story reinforced concrete building, Part II: observed wave numbers. Soil Dyn Earthquake Eng 2001;21(3):211-23.
[12] Trifunac MD, Ivanovic SS, Todorovska MI. Wave propagation in a seven-story reinforced concrete building, III: damage detection via changes in wave numbers. Soil Dyn Earthquake Eng 2001; 23(1):65-75.

[13] Todorovska MI, Trifunac MD, Lee VW. Investigation of earthquake response of long buildings. Report No. CE 88-02, Department of Civil Engineering, University of Southern California, Los Angeles, California; 1988 (can be downloaded from: $\langle$ http://www.usc.edu/ dept/civil_eng/Earthquake_eng/ $>$ ).

[14] Todorovska MI, Hayir A, Trifunac MD. Antiplane response of a dike on flexible embedded foundation to incident SH-waves. Soil Dyn and Earthquake Eng 2001;21(7):593-601.

[15] Todorovska MI, Trifunac MD. Antiplane earthquake waves in long structures. J Eng Mech ASCE 1989;115(12):2687-708.

[16] Todorovska MI, Trifunac MD. A note on the propagation of earthquake waves in buildings with soft first floor. J Eng Mech ASCE 1990;116(4):892-900.

[17] Todorovska MI, Trifunac MD. A note on excitation of long structures by ground waves. J Eng Mech ASCE 1990;116(4):952-64.

[18] Todorovska MI, Lee VW. Seismic waves in buildings with shear walls or central core. J Eng Mech ASCE 1989;115(12):2669-86.

[19] Safak E. New approach to analyzing soil-building systems. Soil Dyn Earthquake Eng 1998;17:509-17.

[20] Gicev V. Investigation of soil-flexible foundation-structure interaction for incident plane SH waves. PhD dissertation, Department of Civil Engineering, University of Southern California, Los Angeles, California; 2005.

[21] Todorovska MI, Trifunac MD. Analytical model for in-plane building-foundation-soil interaction: incident P-, SV- and Rayleigh waves. Report No. 90-01, Department of Civil Engineering, University of Southern California, Los Angeles, California; 1990 (can be downloaded from: 〈http://www.usc.edu/dept/civil_eng/ Earthquake_eng/ $>$ ).

[22] Lee VW. Investigation of three-dimensional soil-structure interaction. Report No. CE 79-11, Department of Civil Engineering, University of Southern California, Los Angeles, California; 1979 (can be downloaded from: 〈http://www.usc.edu/dept/civil_eng/ Earthquake_eng/ $>$ ).

[23] Husid R. Gravity effects on the earthquake response of yielding structures. PhD thesis, California Institute of Technology, Pasadena, California; 1967.

[24] Todorovska MI, Trifunac MD. Radiation damping during twodimensional building-soil interaction. Report No. 91-01, Department of Civil Engineering, University of Southern California, Los Angeles, California; 1991 (can be downloaded from: 〈http://www.usc.edu/ dept/civil_eng/Earthquake_eng/ $>$ ).

[25] Todorovska MI, Trifunac MD. The effects of wave passage on the response of base-isolated buildings on rigid embedded foundations. Report No. CE 93-10, Department of Civil Engineering, University of Southern California, Los Angeles, California; 1993 (can be downloaded from: 〈http://www.usc.edu/dept/civil_eng/Earthquake_ eng/ $>$ ).

[26] Dablain MA. The application of high order differencing to the shear wave equation. Geophysics 1986;51(1):54-66.

[27] Lax PD, Wendroff B. Difference schemes for hyperbolic equations with high order of accuracy. Comm on Pure Appl Math 1964;XVII:381-98.

[28] Fujino Y, Hakuno M. Characteristics of elasto-plastic ground motion during an earthquake. Bull Earthquake Res Inst Tokyo Univ 1978;53:359-78. 